\title{
Aufsätze
}

\section{Herennius Dexippus und die Tradition der Perserkriege im Imperium Romanum}

von Robert Rollinger

I.

Die Entdeckung eines neuen Fragments der Skythika des im 3.Jahrhundert n. Chr. schreibenden griechischen Historiographen Herennius Dexippus vor wenigen Jahren erfuhr in der Fachwelt ein großes Echo. ${ }^{\mathrm{I}}$ Auch wenn sich die Autorenschaft des

I Caillan Davenport/Christopher Mallan, Dexippus' Letter of Decius. Context and Interpretation, in: Museum Helveticum 70, 2013, 57-73; Jana Grusková/Gunther Martin, Ein neues Textstück aus den ,Scythica Vindobonensia‘ zu den Ereignissen nach der Eroberung von Philippopolis, in: Tyche 29, 20I4, 29-43; dies., Zum Angriff der Goten unter Kniva auf eine thrakische Stadt (Scythica Vindobonensia,f. I 95 ${ }^{\mathrm{v}}$ ), in: Tyche 30, 20I 5, 35-53; dies., Rückkehr zu den Thermopylen. Die Fortsetzung einer Erfolgsgeschichte in den neuen Fragmenten Dexipps von Athen, in: Armin Eich/Stefan Freund/Meike Rühl/Christoph Schubert (Hrsg.), Das dritte Jahrhundert. Kontinuitäten, Brüche, Übergänge. (Palingenesia, I08.) Stuttgart 20I7, 267-28I; dies., Neugelesener Text im Wiener Dexipp-Palimpsest (Scythica Vindobonensia,f. I95 ${ }^{\text {v }, ~ Z . ~ 6-I o) ~ m i t ~ H i l f e ~}$ der Röntgenfluoreszenzanalyse, in: ZPE 206, 20I 7, 40-46; Christopher Mallan/Caillan Davenport, Dexippus and the Gothic Invasions. Interpreting the New Vienna Fragment (Codex Vindobonensis Hist. gr. 73 , ff. I $92^{\mathrm{v}}-$ I93 ${ }^{\mathrm{r}}$ ), in: JRS I05, 2015, 203-226; Gunther Martin/Jana Grusková, „Dexippus Vindobonensis“(?). Ein neues Handschriftenfragment zum sog. Herulereinfall der Jahre 267/268, in: Wiener Studien I27, 20 I4, IOI-I 20; dies., ,Scythica Vindobonensia‘ by Dexippus (?). New Fragments on Decius' Gothic Wars, in: GRBS 54, 20I4, 728-754; Ioan Piso, Bemerkungen zu Dexippus Vindobonensis (I), in: Göttinger Forum für Altertumswissenschaft I8, 2015, I99-2 I5; Carlo Martino Lucarini, Zum neuen Dexipp, in: ZPE I97, 2016, 42-45; Gunther Martin, Die Struktur von Dexipps Skythika und die Historia Augusta, in: Bruno Bleckmann/Hartwin Brandt (Eds.), Historiae Augustae Colloquium Dusseldorpiense. Bari 2017, 97-I I4; Giuseppe Zecchini, Il nuovo Dexippo e l'Historia Augusta, in: Bleckmann/Brandt (Eds.), Historiae Augustae Colloquium Dusseldorpiense, I 89-222; Jack W. G. Schropp/Robert Rollinger, Exercitus Romanus ad Thermopylas? Zuf. I94 ${ }^{\mathrm{r}}$ Z. I-I 6 im neuen 
Herennius nicht mit allerletzter Sicherheit beweisen lässt, so ist sie doch als überaus wahrscheinlich anzunehmen. ${ }^{2}$ Obwohl es auch bei der historischen Einordnung der in dem Fragment beschriebenen Ereignisse gewisse Unwägbarkeiten gibt, so spricht doch einiges dafür, dass darin ein Goteneinfall in den beginnenden sechziger Jahren des 3.Jahrhunderts ziemlich detailreich geschildert wird. ${ }^{3}$ Hat sich die Forschung bisher stark auf die philologische und historische Erschließung des Fragments konzentriert ${ }^{4}$, so blieb ein rezeptionsgeschichtlicher Aspekt bisher einigermaßen unbeleuchtet ${ }^{5}$. Dabei geht es um den Stellenwert der Perserkriege im literarischen Diskurs des Imperium Romanum, auf den das Dexippus-Fragment ein neues Schlaglicht wirft. Dieser Aspekt soll im Zentrum der folgenden Betrachtungen stehen.

Dexipp, in: Kai Ruffing/Kerstin Droß-Krüpe (Hrsg.) Emas non quod opus est, sed quod necesse est. Studien zur Wirtschafts-, Sozial-, Wissenschafts- und Rezeptionsgeschichte der Antike. Festschrift für Hans-Joachim Drexhage zum 70. Geburtstag. Wiesbaden 2018, 429-438. Siehe auch Christopher P. Jones, Further Fragments of Dexippus, URL: https://www.academia.edu/I I 9I3736/Further_Dexippus_online (27.I 2.20I 7); ders., Further Fragments of Dexippus (2), URL: https://www.academia.edu/26I9904I/Further_Dexippus_2_ (29.I.20I8).

2 Mallan/Davenport, Gothic Invasions (wie Anm. I), 207-2 Io; Martin/Grusková, Herulereinfall (wie Anm. I), I I3-I I 6; dies., Goten (wie Anm. I), 48-50; Martin, Struktur (wie Anm. I), 97 f. Zum Autor und zu den bisher, also vor der Entdeckung des ,Wiener Dexipp‘, bekannten Fragmenten siehe Gunther Martin, Dexipp von Athen. Edition, Übersetzung und begleitende Studien. (Classica Monacensia, 32.) Tübingen 2006; Laura Mecella, Dexippo di Atene. Testimonianze e frammenti. Tivoli $20{ }_{3}$.

3 Die Datierung des Ereignisses ist umstritten. Für einen Ansatz ins Jahr 262 n. Chr. plädieren durchaus überzeugend Mallan/Davenport, Gothic Invasions (wie Anm. I), 2 I5-220; ebenso mit weiteren Argumenten Piso, Bemerkungen (wie Anm. I), I99-206. Demgegenüber plädieren Jones, Further Fragments (wie Anm. I), 4f., Jones, Further Fragments (2) (wie Anm. I), 4f., Bruno Bleckmann, Südosteuropa im III. und IV. Jahrhundert - ereignisgeschichtlicher Teil. Version: I.o, in: Online-Handbuch zur Geschichte Südosteuropas. Bd. I: Herrschaft und Politik in Südosteuropa bis I 8oo. Hrsg. vom Institut für Ost- und Südosteuropaforschung, 3.5.20I6, URL: http://www.hgsoe.ios-regensburg.de/themen/herrschaft-politik-und-staatlichkeit.html (29.I.20I 8), gf., Lucarini, Neuer Dexipp (wie Anm. I), 45, und Martin, Struktur (wie Anm. I), Io6, für eine Datierung der Ereignisse in das Jahr 253/54 n.Chr. (vgl. auch ebd. I I 3 Anm. 65). Zum allgemeinen historischen Kontext vgl. Roland Steinacher, Rom und die Barbaren. Völker im Alpen- und Donauraum (300600), Stuttgart 20I7, 5 If.

4 Siehe Anm. I.

5 Dazu jetzt aber Grusková/Martin, Thermopylen (wie Anm. I). 
Das fragliche Fragment (Codex Vindobonensis historicus gr. 73, ff. $\left.192^{\mathrm{v}}-193^{\mathrm{r}}\right)^{6}$ nennt im Verlauf der Ereignisse drei Personen in militärischen Leitungsfunktionen, die dabei sind, an den Thermopylen ${ }^{7}$ eine Verteidigungsstellung einzurichten, um sich auf die anstehende Auseinandersetzung mit dem als Skythen bezeichneten Gotenverband vorzubereiten. ${ }^{8}$ Die ,Barbaren' hatten nach den vorangehenden Ausführungen des Textes zuvor erfolglos Thessaloniki belagert und waren dann brandschatzend weiter nach Süden gezogen, in der Hoffnung auf reiche Beute in den Städten und Tempeln der römischen Provinzen Macedonia und Achaea. Das Kommando am Thermopylenpass hat offensichtlich der Römer Marianus inne, dem neben den beiden lokalen Kommandeuren, dem Böotarchen Dexippus und einem gewissen Philostratos aus Athen ${ }^{9}$, vor allem ein von der Provinz gestelltes Aufgebot untersteht, das vornehmlich aus der lokalen griechischsprachigen Bevölkerung besteht ${ }^{\text {IO }}$. Der vom Kaiser eingesetzte Marianus dürfte wohl als Statthalter der Provinz Achaea anzusprechen sein. ${ }^{\text {II }}$

Es ist nun auch dieser Marianus, der vor dem versammelten Aufgebot seiner griechischen Soldaten eine packende Rede hält, die nicht zuletzt darauf abzielt, den Kampfesmut seiner Truppe anzufachen und sie auf die bevorstehende Auseinander-

\footnotetext{
6 Ebd.28of. Vgl. auch Martin/Grusková, Herulereinfall (wie Anm. I), I I3-I20; Mallan/Davenport, Gothic Invasions (wie Anm.I), $205 \mathrm{f}$.

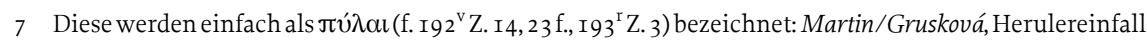
(wie Anm. I), Io7 f. Sie werden auchf. I $94^{\mathrm{r}}{ }^{\mathrm{Z}}$ Z. IO, I5 genannt, als dort von Athenern, Böotern und Spartanern eine Verteidigungsstellung errichtet wird; Grusková/Martin, Neues Textstück (wie Anm. I), 32 f. Dieses Ereignis ist wohl in das Jahr 250 n. Chr. zu datieren; ebd. 30, 37-43.

8 Text und Übersetzung des gesamten Abschnitts bei Martin/Grusková, Herulereinfall (wie Anm. I), Io6Io9; Mallan/Davenport, Gothic Invasions (wie Anm. I), 205 f.

9 Zum Böotarchen Dexippus und zu Philostratos aus Athen siehe Mallan/Davenport, Gothic Invasions (wie Anm. I), 2I 2-2 I4. Der Böotarch ist nicht mit dem Autor des Fragments identisch.

Io Wie folio I92 ${ }^{\mathrm{v}}$ lehrt, waren diese, wie es sich für ein Volksaufgebot gehört, nur notdürftig bewaffnet.

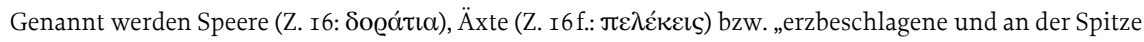

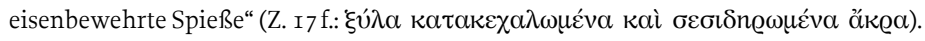

I I Mallan/Davenport, Gothic Invasions (wie Anm. I), 2 Io-2 I 2. Nach Piso, Bemerkungen (wie Anm. I), 2 I 2-2 I 4, sei Marianus als ein agens vice praesidis provinciae Achaiae anzusprechen, ein erfahrener ritterlicher Offizier, der den Prokonsul ersetzte und neben dem Volksaufgebot auch einen Legionsverband befehligte. Dexipp habe die römischen Soldaten bewusst nicht genannt, um so „eine Geschichte zu schreiben, in welcher den Griechen und nicht den Römern eine zentrale Rolle eingeräumt wird“; ebd. 208f.
} 
setzung vorzubereiten. Es mag in diesem Zusammenhang nicht überraschen, dass Marianus dabei im Besonderen auf den Genius Loci der Thermopylen und die Bedeutung dieses Ortes in jenen militärischen Auseinandersetzungen hinweist, in denen

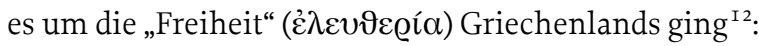

„Griechen! Der Beweggrund, aus dem ihr euch versammelt habt - unsere Rettung -, und die Gegend, in der ihr euch aufgestellt habt, sind beide in hohem Maße dazu angetan, die Erinnerung an vollbrachte Leistungen zu erwecken. Denn auch eure Vorfahren haben, wenn sie an diesem Ort kämpften, nie den Kampf um die Freiheit Grie-

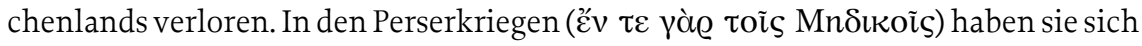
nämlich auf das Beste geschlagen und im sogenannten Lamischen Krieg und auch dann wieder, als sie Antiochos, den Herrscher Asiens, in die Flucht schlugen, schon auf Seiten der damals führenden Römer. Gewissermaßen wie vom Schicksal, nach dem Willen irgendeiner Gottheit, war und ist den Griechen an diesem Ort in den Kämpfen gegen die Barbaren Glück zugewiesen. Eigene militärische Vorhaben sind bei euch lange Zeit nicht vorgekommen. Doch ihr könnt nun Zuversicht schöpfen einerseits aus eurer eigenen Heeresmacht, andererseits aus der natürlichen Befestigung des Geländes, durch die ihr euch den Gegnern auch bei den früheren Angriffen als Furcht erweckend erwiesen habt. Aus diesen Gründen ist meiner Ansicht nach auch die Zukunft keineswegs hoffnungslos, da ich gut weiß, dass [ ... ] zum besseren [... ].“ ${ }^{13}$

Auf den ersten Blick überraschen die historischen Vergleichsbeispiele, derer sich Marianus in seiner Rede bedient. Einem modernen Leser kämen als erstes wohl sofort die Perserkriege des 5.Jahrhunderts v.Chr. in den Sinn, sodass die Verweise auf den Lamischen Krieg (323-322 v.Chr.) und den Syrischen Krieg (I92-I88 v.Chr.) verwundern mögen. ${ }^{\text {I4 }}$ Allerdings liegt genau in dieser speziellen Vergleichskonstruktion der Quellenstelle ihr besonderer Wert, ruft sie doch an dem auch in der

I2 Codex Vindobonensis historicus gr. 73, palimpsestierte folio I93 ${ }^{\mathrm{r}}$, Z. I4-30: Grusková/Martin, Thermopylen (wie Anm. I), 28I (Transliteration); Mallan/Davenport, Gothic Invasions (wie Anm. I), 205f. (Transkription).

I3 Übersetzung nach Grusková/Martin, Thermopylen (wie Anm. I), 269; vgl. auch Mallan/Davenport, Gothic Invasions (wie Anm. I), 204.

I Karl-Wilhelm Welwei, s. v. Thermopylai, in: Encyclopedia of Ancient History. Malden 2013, 6704-6705, etwa erwähnt als einzige ,Schlacht‘ an den Thermopylen nur jene der Perserkriege; Ernst Meyer/Gerhard Dohrn-van Rossum, s. v. Thermopylai, in: Der Neue Pauly I2/I, 2002, 427-43 I, bieten demgegenüber ein wesentlich breiteres Panorama. 
römischen Kaiserzeit instrumentalisierbaren Erinnerungsort der Thermopylen historische Konnotationen auf, die als zeitgenössische Erinnerungsräume zu bewerten sind. Damit wird das Dexippus-Fragment auch zu einem wichtigen Zeugnis für diese Erinnerungshorizonte sowie für deren Gewichtung und Instrumentalisierung in ihrem spezifischen zeitgeschichtlichen Kontext. Es gilt sowohl die strukturell-literarische Dimension des Fragments als auch die weiteren zeitgeschichtlichen Kontexte aufzuspüren und in einen größeren historischen Bezugsrahmen zu stellen. Was die literarische Analyse des Fragments und seinen Aufbau in Bezug auf die Thermopylenschlacht betrifft, haben unlängst Jana Grusková und Gunther Martin grundlegende Vorarbeiten geleistet. ${ }^{15}$ Eine Beobachtung der beiden Autoren verdient in diesem Zusammenhang besonderes Interesse.

So machten sie zunächst darauf aufmerksam, dass die angeführten Vergleichsbeispiele bei näherer Betrachtung eigentlich nicht besonders stimmig sind. ${ }^{16}$ Diese Unstimmigkeiten lösen sich allerdings auf, wenn die Wirkung der Rede sowohl in ihrer an die griechischen Soldaten gerichteten intra-narrativen als auch in ihrer an den des Griechischen mächtigen Leser/Zuhörer gerichteten extra-narrativen Dimension nicht primär auf die Frage von Erfolg oder Misserfolg des zu erwartenden Kampfes abzielt, sondern die Fokussierung auf die heldenhafte Tat im Kontext heroischer Ereignisse der Vergangenheit im Auge hat. Bezeichnenderweise appelliert Marianus zu Beginn seiner Rede „an die Erinnerung tugendhaften Handelns“

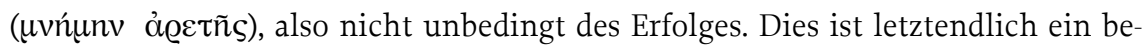
kanntes Muster der Exhortation und Selbstvergewisserung, das in der Weltgeschichte immer wieder begegnet. So kann der Verweis auf eine verlorene Schlacht, ist sie in einem allgemeineren Bewusstsein im Sinne einer als ,gerecht' oder ,national' erachteten Sache geführt worden, nicht nur sinnstiftend sein, sondern auch

\footnotetext{
I5 Grusková/Martin, Thermopylen (wie Anm. I).

I6 Ebd. 272-274. So mögen sich im Lamischen Krieg die sich gegen die Makedonen erhebenden griechischen Truppen zwar bei den Thermopylen verschanzt haben, doch wurde die eigentliche Schlacht beim benachbarten Herakleia geschlagen. Immerhin war diese aber für die Verteidiger erfolgreich, auch wenn sie letztlich den Krieg gegen die von Antipater geführten übermächtigen Makedonen verloren. Im Falle des Syrischen Krieges konnten sich die Verteidiger an den Thermopylen allerdings keineswegs erfolgreich behaupten. Vielmehr umgingen die von M. Acilius Glabrio angeführten römischen Truppen den Pass und schlugen Antiochos III. und seine Verbündeten in die Flucht, für den der Krieg letztendlich auch mit einer vernichtenden Niederlage endete. Durchaus Vergleichbares hatte sich ja schon während der Perserkriege ereignet. Auch hier waren die Verteidiger an den Thermopylen keineswegs erfolgreich, auch wenn man schließlich die Perser aus Griechenland vertreiben konnte.
} 
trefflich zur Mobilisierung dienen. Dies zeigen ja nicht nur bekannte Beispiele wie etwa die Schlacht auf dem Amselfeld (I389) und ihre Bedeutung für die Etablierung einer südslawisch-serbischen Identität, sondern gerade auch die westliche Rezeption der Thermopylenschlacht der Perserkriege seit dem I8.Jahrhundert. Trotz der Tatsache, dass es sich bei dem Scharmützel an den Thermopylen eigentlich um einen militärisch erfolglosen und strategisch wohl auch völlig sinnlosen Abwehrkampf handelte ${ }^{17}$, wurde das Ereignis in der Folge zu einem heroischen Spektakel aufgeblasen, das selbst Hermann Göring noch dazu diente, dem sich abzeichnenden Debakel der in Stalingrad eingeschlossenen Verbände der Wehrmacht einen Sinn zu geben ${ }^{\mathrm{I} 8}$.

Funktionieren konnte das freilich nur, weil man die vom Hellenenbund erfochtenen ,Siege‘ der Perserkriege in einem eurozentristischen Blickwinkel als ,welthistorische Siege' und demzufolge als epochale Ereignisse deutete. ${ }^{19}$ Man kämpfte also für eine ,gerechte Sache', für die ,Freiheit‘, was immer man auch darunter verstand. ${ }^{20}$ Unter diesem Blickwinkel betrachtet lösen sich die von Grusková und Martin vorgebrachten Bedenken, was die mögliche Validität der historischen Vergleichsbeispiele betrifft, sofort auf. Diese gewinnen vielmehr an Struktur und Kontur und eröffnen einen sowohl sinnvollen als auch stimmigen Erklärungshorizont. Es geht um den in einer Gemeinschaft geführten heroischen Kampf gegen barbarische Inva-

I7 Hier konnten auch die scharfsinnigen Worte Belochs wenig ausrichten; Karl Julius Beloch, Griechische Geschichte. Bd. 2/2: Bis auf die sophistische Bewegung und den Peloponnesischen Krieg. 2.Aufl. Straßburg I9I6, I04f. Vgl. Stefan Rebenich, From Thermopylae to Stalingrad. The Myth of Leonidas in German Historiography, in: Anton Powell/Stephen Hodkinson(Eds.), Sparta. Beyond the Mirage. London/Swansea 2002, 326f.; Anuschka Albertz, Exemplarisches Heldentum. Die Rezeptionsgeschichte der Schlacht an den Thermopylen von der Antike bis zur Gegenwart. München 2006, 250-260.

I8 Albertz, Heldentum (wie Anm. I7), 293-308.

I9 Grundlegend dazu:Josef Wiesehöfer, „Denn es sind welthistorische Siege ..." Nineteenth- and TwentiethCentury German Views on the Persian Wars, in: History \& Culture II, I992, 6I-83; ders., „Griechenland wäre unter persische Herrschaft geraten...“ Die Perserkriege als Zeitenwende?, in: Sven Sellmer/Horst Brinkhaus (Hrsg.), Zeitenwenden. Historische Brüche in asiatischen und afrikanischen Gesellschaften. (Asien und Afrika, 4.) Hamburg 2002, 2 I I-232.

20 Zum Freiheitsbegriff in der Klassischen Antike vgl. grundlegend Kurt Raaflaub, Die Entdeckung der Freiheit. Zur historischen Semantik und Gesellschaftsgeschichte eines politischen Grundbegriffs der Griechen. (Vestigia, 37.) München I985; ders., Zwischen Adel und Volk. Freiheit als Sinnkonzept in Griechenland und Rom, in: Karl-Joachim Hölkeskamp/Jörn Rüsen/Elke Stein-Hölkeskamp/Heinrich Theodor Grütter (Hrsg.), Sinn (in) der Antike. Orientierungssysteme, Leitbilder und Wertkonzepte im Altertum. Mainz 2003, 55-80. 
soren aus dem Norden, denen man sich mit Kampfesmut und im Bewusstsein einer gerechten Sache entgegenzustellen hat. Bemerkenswert ist dabei eher, dass Perser, Makedonen, ,Seleukiden` und Skythen (Goten) auf eine ähnliche Stufe gestellt und als von außen kommende feindliche Horden dargestellt werden können. Dieser Aspekt verdient besondere Beachtung. Dabei kommen wir langsam einem zeitgenössischen Kontext des von Dexippus entworfenen Geschichtsbildes auf die Spur, den Grusková und Martin nur in Ansätzen berührt haben.

Zwei Dinge springen in diesem Zusammenhang ins Auge. Zunächst erhält Marianus' Rede dadurch eine besondere Note, dass sich sein Appell nicht an Bewohner selbständiger griechischer Poleis richtet, sondern an römische Provinzbewohner, über die er als Römer das Kommando und damit die Befehlsgewalt ausübt. ${ }^{2 \mathrm{I}}$ Dies schafft gewisse Unschärfen zu den angeführten Vergleichsbeispielen auf zwei Ebenen, wobei sich Marianus expressis verbis nur mit einer von beiden auseinandersetzt. Der erste nur indirekt angesprochene Aspekt betrifft die tiefe chronologische und historische Distanz zu den Vergleichsbeispielen aus der Geschichte. Sie wird durch ein Kontinuitätskonstrukt überbrückt, indem Marianus seine ihm an den Thermopylen unterstehenden Provinzbewohner einfach als Hellènes anspricht und sie somit stillschweigend mit den historischen Protagonisten gleichsetzt. ${ }^{22}$ Der zweite Aspekt wird demgegenüber offen thematisiert. Der Umstand, dass die griechischsprachige Provinzbevölkerung eigentlich schon lange nicht mehr selbst zu den Waffen greifen musste, sondern vielmehr unter dem Dach der pax Romana ein gedeihliches Auskommen fand, war im gegebenen Zusammenhang insofern ein Problem, als Kriegserfahrung und Wehrtüchtigkeit notgedrungen unter der inzwischen mehrere Jahrhunderte dauernden Friedenszeit leiden mussten. Marianus verschleiert diesen Sachverhalt keineswegs, sondern spricht ihn direkt an: „Eigene mi-

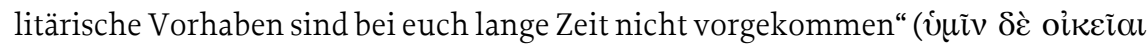

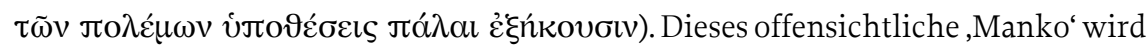
in den noch erhaltenen Teilen der Rede des Marianus durch den Hinweis auf den besonderen Charakter der gewählten Verteidigungsstellung auszuräumen versucht. Damit wird prinzipiell wohl nicht nur auf die für die Verteidiger militärtechnisch äußerst günstige Geländesituation verwiesen, sondern auch auf den Genius Loci, womit erneut die historischen Vergleichsbeispiele ins Bewusstsein gerufen werden.

2I Grusková/Martin, Thermopylen (wie Anm. I), 274.

22 Ebd. $267-272$. 
Die Tatsache, dass die anstehende Auseinandersetzung mit den Skythen (Goten) unter römischem Kommando geführt wird, ist ein zentrales Moment der Rede des Marianus und des in diesem Zusammenhang entworfenen Geschichtsbildes, das eine eingehende Betrachtung verdient. Denn wäre Marianus wirklich primär daran gelegen gewesen, die ihm unterstehenden und als „Griechen“ angesprochenen Truppen in ihrer „griechischen“ Identität zu bekräftigen, hätte der Hinweis auf die „Perserkriege“ als Analogon vollauf genügt. Hier hatte eine Koalition freier griechischer Poleis gegen eine von Persern geführte Invasionsarmee gekämpft und die Eindringlinge letztendlich erfolgreich aus Griechenland vertrieben. Die geschichtsbewussten Böoter unter Marianus' Soldaten hätten dabei zwar etwas die Nase gerümpft, fochten sie doch auf persischer Seite, letztendlich verschwanden die Perser aber auch aus ihrem Land. Der Lamische Krieg, in dem die Böoter auf makedonischer Seite fochten ${ }^{23}$, war diesbezüglich als Vergleichsbeispiel auf jeden Fall problematisch, handelte es sich doch eigentlich um einen letztendlich gescheiterten Aufstand einzelner griechischer Poleis gegen die makedonischen Oberherren. Noch problematischer war der Vergleich mit dem Syrischen Krieg, denn hier focht Athen nicht nur auf der Seite des Seleukiden, sondern die griechischen Poleis waren in dieser Auseinandersetzung eigentlich nur der Spielball zwischen zwei Großmächten, von denen sich letztlich Rom durchsetzte. Und doch ist es dieses Vergleichsbeispiel, dem in dem von Marianus entworfenen Geschichtsbild die entscheidende Rolle zu-

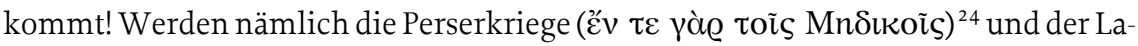
mische Krieg eigentlich nur en passant erwähnt, so wird der gegen Antiochos III. ausgefochtene Syrische Krieg mit zwar wenigen, aber doch gewichtigen Erläuterungen in einen besonderen Sinnzusammenhang gestellt. So wird Antiochos als „Herr-

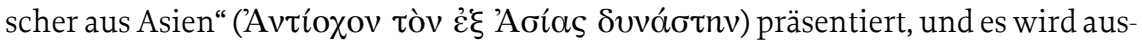
drücklich hervorgehoben, dass die gegen den Seleukiden kämpfenden Griechen

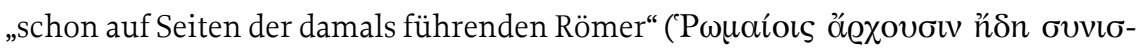
тóucvor) ins Feld gezogen waren. Damit wird eine klare Gewichtung der Vergleichsbeispiele vorgenommen. Es mag durchaus stimmen, dass sich Dexippus mit seiner

23 Martin Jehne, Koine Eirene. Untersuchungen zu den Befriedungs- und Stabilisierungsbemühungen in der griechischen Poliswelt des 4.Jahrhunderts v. Chr. (Hermes Einzelschriften, Bd. 63.) Stuttgart I994, 264. 24 Zur Bezeichnung der Perserkriege als „Mederkriege“vgl. Christopher J. Tuplin, Persians as Medes, in: Heleen Sancisi-Weerdenburg/Amélie Kuhrt/Margaret Cool Root (Eds.), Continuity and Change. (Achaemenid History, Vol. 8.) Ann Arbor/Leiden I994, 235-256. 
Referenz auf die Perserkriege, den Lamischen Krieg und die römischen Feldzüge in Griechenland in eine Tradition einreiht, „die von Herodot über Ephoros bis zu Polybios reicht“ ${ }^{25}$, doch erklärt dies keineswegs die unterschiedlichen Gewichtungen des angestellten Vergleichs, bei dem den Perserkriegen und dem Lamischen Krieg bestenfalls eine untergeordnete Rolle zukommt.

Es ist nicht nur die einzig am Beispiel des Syrischen Krieges zum Ausdruck kommende „Kampfgemeinschaft' zwischen Griechen und Römern, die dieser spezifischen Auseinandersetzung im Vergleich zu den anderen beiden Beispielen einen besonderen Stellenwert einräumt. Dabei spielen auch wirkmächtige Erzähltraditionen eine wichtige Rolle. Wie wir bereits beobachten konnten, sind bei der Qualifizierung des jeweiligen Gegners deutliche Unterschiede festzustellen. So bleibt der Lamische Krieg diesbezüglich als einziges Beispiel völlig vage, während für das Vergleichsbeispiel,Perserkriege im Gegensatz dazu zumindest die elementarsten Informationen geliefert werden: Es waren Perser (Meder), gegen die damals an den Thermopylen gefochten wurde. Einmal mehr sticht der Syrienkrieg im Vergleich dazu deutlich hervor, wird doch nur hier eine wirkmächtige Zusatzinformation geliefert, die zwar in der konkreten Fassbarkeit des Gegners vage, in ihrem durch die Tradition vorgegebenen Erklärungshorizont jedoch ziemlich konkret bleibt: Damals wurde gemeinsam gegen Asiaten gefochten. Damit wird indirekt auf die mindestens seit dem 5.Jahrhundert v.Chr. wirksame Dichotomie zwischen Europa und Asien angespielt, und es ist durchaus bemerkenswert, dass die entsprechenden Konnotationen durch den Syrischen Krieg und nicht durch die Perserkriege abgerufen werden. Lässt man diese unterschiedlichen Gewichtungen Revue passieren, ergibt sich eine klare Hierarchisierung der Vergleichsbeispiele. Die wirkungsmächtigste und in den Augen des Marianus schlagendste Parallele wird durch den Syrischen Krieg abgerufen; darauf folgen die Perserkriege, die, gemeinsam mit ersterem, den Lamischen Krieg, das - wohl nicht nur in den Augen des modernen Betrachters - am wenigsten zugkräftige Vergleichsbeispiel rahmen. In diesem Zusammenhang taucht sofort die Frage auf, ob es sich dabei um ein völlig singuläres Geschichtsbild handelt, das Dexippus Marianus im vorliegenden Kontext entwerfen lässt, oder ob sich die hier deutlich werdenden Vorstellungswelten in einen größeren Rahmen stellen lassen.

25 Grusková/Martin, Thermopylen (wie Anm. I), 275. 
III.

Zuletzt haben sich mehrere Autoren eingehender mit der Tradition der Perserkriege im Imperium Romanum beschäftigt. ${ }^{26}$ Dabei wurde meist generell, entweder explizit oder implizit, postuliert, dass die römischen mit den griechischen Eliten in spätrepublikanischer Zeit sowie in der frühen Kaiserzeit des I.Jahrhunderts n.Chr. eine in gewisser Hinsicht gemeinsame Erinnerungskultur („shared memory“) geteilt hätten und somit auch etwas von der griechischen „Persian-wars ,mania““ in den römischen Quellen dieser Zeit aufzuspüren sei. ${ }^{27}$ Gleichzeitig wird dieser angebliche gemeinsame Erinnerungshorizont als Teil einer quasi offiziellen Reichsideologie interpretiert ${ }^{28}$, wobei Rom als Nachfolgerin des Hellenenbundes und die Parther als Erben der Perser/Achaimeniden präsentiert worden seien. Freilich bot sich spätestens seit der Schlacht von Carrhae (53 v.Chr.) die Gelegenheit, den Erinnerungshorizont der Perserkriege in einen neuen Kontext zu stellen und als universellen Kampf gegen die östlichen Barbaren, in diesem Fall die Parther, zu verste-

26 Antony Spawforth, Symbol of Unity? The Persian-Wars Tradition in the Roman Empire, in: Simon Hornblower (Ed.), Greek Historiography. Oxford I994, 233-247; Philip Hardie, Images of the Persian Wars in Rome, in: Emma Bridges/Edith Hall/Peter Jones Rhodes (Eds.), Cultural Responses to the Persian Wars. Antiquity to Third Millennium. Oxford 2007, I27-I43; Charlotte Lerouge, L'image des Parthes dans le monde gréco-romain. Du début du rer siècle av. J.-C. jusqu'à la fin du Haut-Empire romain. (Oriens et Occidens, I7.) Stuttgart 2007, I24-I27, I47-I49, I63f., I67; Aleksandr V. Makhlaiuk, Memory and Images of Achaemenid Persia in the Roman Empire, in: Jason M. Silverman/Caroline Waerzeggers (Eds.), Political Memory in and after the Persian Empire. Atlanta 2015, 299-324; Emma Bridges, Imagining Xerxes. Ancient Perspectives on a Persian King. London/New Delhi/New York/Sydney 2015; Eran Almagor, The Empire Brought Back: Persianism in Imperial Greek Literature, in: Rolf Strootman/Miguel John Versluys (Eds.), Persianism in Antiquity. (Oriens et Occidens, 25.) Stuttgart 2017, 327-343.

27 Die Zitate stammen von Spawforth, Unity (wie Anm. 26), 233.

28 Laut Susan E. Alcock, Archaeologies of the Greek Past. Landscape, Monuments, and Memories. Cambridge 2002, 82 f., seien die Perserkriege „a shared symbol between Greeks and their Roman masters“. Ähnlich auch Hardie, Images (wie Anm. 26), I36, der festhält: „the anology of the Greek defeat of the Persians became embedded in the Roman consciousness as a defining element of national identity". Differenzierter zunächst Makhlaiuk, Memory (wie Anm. 26), 305ः „Of course, these wars, and Xerxes's invasion above all, were not a subject of interest per se for the Romans, and they did not have the same emotional resonance as for the Greeks. Nevertheless, this was one of many literary themes which learned Romans sought to emulate.“Wenig später allerdings wird, basierend auf der Auseinandersetzung mit den Parthern eine „common, unified Greco-Roman identity“ postuliert und betont: „Consciously or spontaneaously, the Romans represented themselves as the ,new Greeks' of 480-479 B.C.E., who advocated Western values against Eastern despotism, defending Europe against Asia“. Vgl. Auch Almagor, Empire (wie Anm. 26), 330. 
hen. ${ }^{29}$ Hier muss allerdings streng zwischen einer ,offiziellen` imperialen Ideologie und jeweils lokalen Erinnerungsbildern griechischsprachiger Eliten unterschieden werden, die ihre Geschichtsvorstellungen bestenfalls in einem schmeichelnd-anbiedernden Ton den jeweiligen Kaisern nahezubringen versuchten ${ }^{30}$, was natürlich von kaiserlicher Seite in jedem einzelnen Fall erst approbiert werden musste. Dazu gehört etwa die Stiftung einer Ehreninschrift für Kaiser Nero am östlichen Architrav des Parthenon in den frühen sechziger Jahren des I.Jahrhunderts n. Chr., die zeitlich sicherlich nicht zufällig mit dem um Armenien ausgefochtenen Partherkrieg des Kaisers korreliert. ${ }^{3 \mathrm{I}}$ Der Stifter Tib. Claudius Novius war ein Angehöriger der athenischen Elite, der auch eine zentrale Funktion im Rahmen des bei Platäa eingerichteten Lokalkultes einnahm. Dort war nach der Schlacht von 479 v.Chr. ein Heiligtum des Zeus Eleutherios eingerichtet worden, zu dessen Ehren man Eleutherische Spiele veranstaltete. Für deren Durchführung war spätestens seit dem frühen 3.Jahr-

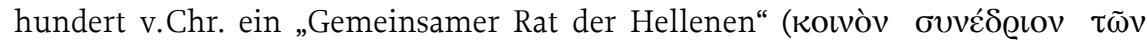

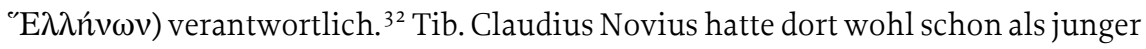

29 Grundlegend zum Partherbild der kaiserzeitlichen Überlieferung ist Lerouge, Parthes (wie Anm. 26). Vgl. in neuerer Zeit auch Erich S. Gruen, Josephus' Image of the Parthians, in: Josef Wiesehöfer/Sabine Müller (Eds.), Parthika. Greek and Roman Authors' Views of the Arsacid Empire. (Classica et Orientalia, I 5.) Wiesbaden 2017, 223-240; ferner in diesem Band die Beiträge von: Sabine Müller, Das Bild der Parther bei Trogus-Justin, 24I-257; Matthäus Heil, Die Parther bei Tacitus, 259-278; Charlotte Lerouge-Cohen, L'image des Parthes chez Arrien. Réflexions sur quelques fragments attribués aux Parthika, 279-305.

30 Dies deutet Spawforth, Unity (wie Anm. 26), 246, immerhin an. Dabei ist unbestritten, dass der Rückgriff auf die Perserkriege wichtig für das Selbstverständnis griechischsprachiger aristokratischer Eliten bestimmter Poleis war; Thomas Schmitz, Bildung und Macht. Zur sozialen und politischen Funktion der zweiten Sophistik in der griechischen Welt der Kaiserzeit. (Zetemata, 97.) München I997, 39-66. So streicht etwa Michael Jung, Marathon und Plataiai. Zwei Perserschlachten als ,lieux de mémoire im antiken Griechenland. (Hypomnemata, I64.) Göttingen 2006, 205-224, die besondere Bedeutung Athens und der festlandgriechischen Poleis in diesem Zusammenhang heraus, postuliert dann aber unversehens, dass „die Vergangenheit der Perserschlacht für eine politische Aktualisierung weiter nutzbar (und sich) auch die römische Politik (ihrer bedient haben soll)“ (ebd.234). Als einziger Beleg dafür dient Jung der von Gordian III. im Jahr 235 n.Chr. in Rom eingerichtete Agon zu Ehren der Athena Promachos (siehe dazu unten Anm. I09).

3I IG ii ${ }^{2}$, 3277; Kevin K. Carroll, The Parthenon Inscription. (Greek, Roman and Byzanztine Monographs, 9.) Durham I982, I I-25; Spawforth, Unity (wie Anm. 26), 234-237.

32 Albert Schachter, Cults of Boiotia. Vol.3: Potnia to Zeus, Cults of Deities Unspecified by Name. London I994, I25-I43; Roland Étienne/Marcel Piérart, Un décret du Koinon des Hellènes à Platées en l'honneur de Glaucon, fils d'Étéoclès, d'Athènes, in: BCH 99, I975, 63-68; Paul Roesch, Note sur la decret des Hellenes en l'honneur de Glaucon, frère de Chrémonidès, in: ZPE I5, I 979, I80-I82; Luisa Prandi, Platea. Momenti e problemi della storia di una polis. Padua I988, I6I-I 73; Jung, Marathon (wie Anm.30), 299-32 I; Klaus Freitag, 
Mann im Waffenlauf reüssiert. ${ }^{33}$ Der mit Zeus Eleutherios verbundene Kult wurde nun sinnfälligerweise mit einem Kult des regierenden römischen Kaisers verbunden, wobei Tib. Claudius Novius u.a. als „Oberpriester des Nero, des Sohnes von Kaiser Claudius, Germanicus' Sohn, und des Zeus Eleutherios der Hellenen und Epimelet der Stadt (scil. Athen)“ figurierte. ${ }^{34}$ Auf diese Weise ließ sich die eigene Vergangenheit trefflich den römischen Oberherren nahebringen und als besonders bedeutend verkaufen.

Wesentlich schwieriger ist es demgegenüber, das Memorieren der Perserkriege als Teil einer kaiserzeitlichen ,Reichsideologie‘ zu fassen. Entsprechende Konnotationen mag das von Rolf Schneider rekonstruierte ,Siegesmonument' transportiert haben, das Augustus nach der Rückgabe der bei Carrhae verlorenen Feldzeichen im Jahre 20 v.Chr. im Apollo-Tempel auf dem Palatin errichten ließ. ${ }^{35}$ Sowohl in seiner rekonstruierten Form als Dreifußmonument als auch durch seinen Aufstellungsort mag es Erinnerungen an die von den Griechen nach der Schlacht von Platäa im Apollo-Heiligtum von Delphi gestiftete Säule, die auf einem Dreifuß stand, wachgerufen haben. ${ }^{36}$ Ein ähnliches Dreifußmonument mag Hadrian im Bezirk des Olympischen Zeus in Athen errichtet haben, das gewisse Verbindungen zu dem von ihm ins Leben gerufenen Panhellenion gehabt haben könnte. ${ }^{37}$ Ein allfälliger Bezug zu den Perser-

s. v. Plataiai, in: Der Neue Pauly 9, 2000, I093f.; Jung, Marathon, 307-3 I I, macht darüber hinaus deutlich, dass sich die Mitglieder dieses Bundes aus der Peloponnes und Mittelgriechenland rekrutierten und der Bund fest in den Kontext einer antimakedonischen Mobilisierung eingebunden war.

33 Spawforth, Unity (wie Anm. 26), 236. Zum Waffenlauf: Jung, Marathon (wie Anm. 30), 345, 348-350.

34 IG ii ${ }^{2}$, I990. Vgl. Daniel J. Geagan, Tiberius Claudius Novius, the Hoplite Generalship and the Epimeliteia of the Free City of Athens, in: AJPh Ioo, I 979, 285; Spawforth, Unity (wie Anm. 26), 235f.; Jung, Marathon (wie Anm.30), 360-362, mit Verweis auf die Bedeutung des Kultes für die griechischsprachigen Führungseliten. 35 Rolf Michael Schneider, Bunte Barbaren. Orientalenstatuen aus farbigem Marmor in der römischen Repräsentationskunst. Worms I986; ders., Die Faszination des Feindes. Bilder der Parther und des Orients in Rom, in: Josef Wiesehöfer (Hrsg.), Das Partherreich und seine Zeugnisse. (Historia-Einzelschriften, Bd. I 22.) Stuttgart I 998, I I I f.; Rolf Michael Schneider, Friend and Foe. The Orient in Rome, in: Vesta Sarkhosh Curtis /Sarah Stewart (Eds.), The Age of the Parthians. (The Idea of Iran, Vol. 2.) London 2007, 70-75; Thomas Schäfer, Spolia et signa. Baupolitik und Reichskultur nach dem Parthererfolg des Augustus. Göttingen I998, 6770. Die von Augustus zurückgewonnenen Feldzeichen wurden bis zur Einweihung des eigentlichen MarsUltor-Tempels auf dem Augustusforum in einem kleinen Tempel für Mars Ultor auf dem Kapitol untergebracht. Dieser hatte wie der wohl um dieselbe Zeit errichtete Tempel für Roma und Augustus auf der Akropolis die Form eines Monopteros. Laut Schäfer, Spolia, seien die Feldzeichen beim Besuch des Augustus in Athen im Jahre I9 v. Chr. zunächst für kurze Zeit ebendort beherbergt worden.

36 Spawforth, Unity (wie Anm. 26), 238.

Ebd.239. Zum Panhellenion siehe die folgenden Ausführungen. 
kriegen bleibt jedenfalls vage. Dies gilt bei näherer Betrachtung auch für andere öffentliche Inszenierungen der römischen Kaiserzeit, auf die wir nun unser Augenmerk lenken wollen.

So dienten als Seeschlachten (vovuaxía) inszenierte Massenspektakel der frühen Kaiserzeit wohl eher der allgemeinen Ergötzung als der ideologischen Mobilisierung. ${ }^{38}$ Dies mag selbst für die von Augustus im Jahre 2 v.Chr. in seinem stagnum Augusti, einem künstlich angelegten See jenseits des Tiber, organisierte Seeschlacht gelten, die der Kaiser nach einer verbreiteten Lehrmeinung als eine Anspielung auf die Seeschlacht von Salamis veranstaltet habe. ${ }^{39}$ Augustus selbst fand in seinen Res Gestae jedenfalls nicht erwähnenswert, dass es sich dabei um die berühmte Seeschlacht gehandelt haben soll. Er spricht lediglich von einem navalis proeli spectaculum bzw. einer vovuaxías ๆća. ${ }^{40}$ Den Zusammenhang mit Salamis erfahren wir lediglich von Ovid ${ }^{4 \mathrm{I}}$ und Cassius Dio. ${ }^{42}$

Augustus' Stillschweigen über den historischen Hintergrund des Spektakels ist auf jeden Fall bemerkenswert, auch wenn dieser bedeutende Sachverhalt von den meisten Gelehrten, die dem Ereignis einen hochideologischen Hintergrund zusprechen, nicht beachtet wird. ${ }^{43}$ Die Naumachie wurde anlässlich der Einweihung des

38 Anders sehen dies Spawforth, Unity (wie Anm. 26), Lerouge, Parthes (wie Anm. 26), Hardie, Images (wie Anm. 26), Makhlaiuk, Memory (wie Anm. 26), die - zumindest wenn es um mit den Perserkriegen verbundene Inszenierungen geht - einen quasi offiziellen propagandistischen Hintergrund vermuten.

39 Grundlegend und kritisch dazu Kathleen M. Coleman, Launching into History. Aquatic Displays in the Early Empire, in: JRS 83, I 993, 48-74.

40 R. Gest. div. Aug. 23. Vgl. dazu den Kommentar von Alison E. Cooley, Res Gestae Divi Augusti. Text, Translation, and Commentary. Cambridge 2009, 209f. Auch Vell. 2,I00,2 erwähnt nur die naumachia, nicht jedoch deren historischen Hintergrund.

4I Ov. ars I,I 7 I-I 72: „Wie war's doch kürzlich, als Caesar in künstlicher Seeschlacht die Flotte / Persiens antreten ließ gegen die Schiffe Athens?“ Dt. Übersetzung nach: Publius Ovidius Naso, Liebeskunst. Lateinisch-deutsch. Hrsg. u. übers. v. Niklas Holzberg. 5. Aufl. Berlin 20I I, I 9.

42 Cass. Dio 55, I0,7: ,... des weiteren wurde an dem Platz, wo heute noch einige Spuren davon gezeigt werden, eine Seeschlacht zwischen Persern und Athenern veranstaltet; diese Bezeichnungen erhielten nämlich damals die beiden Seestreitkräfte, und wie in alter Zeit gingen die ,Athener‘ als Sieger hervor“; dt. Übersetzung nach Cassius Dio, Römische Geschichte. Übers. v. Otto Veh. 5 Bde. Düsseldorf 2007, hier Bd. 4, 2 I I f. Dazu Kathleen M. Coleman, Fatal Charades. Roman Executions Staged as Mythological Enactments, in: JRS 80, I990, 7I; Coleman, Launching into History (wie Anm.39), 54.

43 Ronald Syme, The Crisis of 2 B.C. (SB der Bayerischen Akad. der Wiss., Philosoph.-hist. Klasse, H. 7.) München I974, I5, war sich dessen freilich vollkommen bewusst, maß diesem Sachverhalt allerdings keine Bedeutung bei: „Augustus in the Res Gestae allots a whole section to the naval battle exhibited in a basin excavated across the Tiber, giving its precise dimensions, the total of the ships of war and the soldiers that 
Mars Ultor-Tempels inszeniert, in den schließlich auch die im Jahre 20 v.Chr. von den Parthern zurückgegebenen Feldzeichen der bei Carrhae untergegangenen Legionen überführt wurden. Es ist wiederum nur Ovid, der explizit einen Zusammenhang mit dem Aufbruch des Augustusenkels Gaius Caesar zu einer Kampagne gegen die Parther herstellt ${ }^{44}$, die dann auch tatsächlich ein Jahr später erfolgte - wenn sich daraus auch letztlich kein römisch-parthischer Krieg entwickelte ${ }^{45}$. Freilich wurde es mit der von Ovid ins Auge gefassten Eroberung des Oriens ultimus nichts. ${ }^{46}$ Bemerkenswert ist auf jeden Fall, dass die Perserkriege bestenfalls indirekt als Bestandteil der offiziellen Propaganda aufblitzen bzw. als Projektionsfolie von der modernen Forschung erschlossen werden. Ähnliches gilt übrigens auch für eine angeblich von Augustus propagierte Gleichsetzung von Actium und Salamis. ${ }^{47}$

Zwei Beobachtungen sind in diesem Zusammenhang von Bedeutung, auch wenn sie von jenem Teil der Forschung, der - nicht nur in der Naumachie des Augustus eine Inszenierung offizieller Reichsideologie im Sinne eines Kampfes gegen die Parther sehen will, ignoriert werden. ${ }^{48}$ So erstaunt es doch einigermaßen, dass man sich bisher nicht gefragt hat, ob die Inszenierung einer Seeschlacht überhaupt als eine passende Reminiszenz auf einen unmittelbar bevorstehenden Partherfeldzug erachtet werden konnte. Wenn die Perserkriege denn als Vergleichsfolie so wichtig gewesen wären, hätte sich doch vielmehr die Inszenierung einer Landschlacht wie etwa Platäa viel besser als Paradigma geeignet, schließlich konnte man kaum erwarten, mit den Parthern ein Seegefecht auszutragen.

manned them (about three thousand). Other sources reveal what is not there stated, a conflict between the navies of Athens and Persia, the former victorious as was intended.“

44 Ov. ars I,I77-228. Vgl. auch Cass. Dio 55,I0,I 8-I9, sowie Cooley, Res Gestae (wie Anm. 40), 2 Io.

45 Lerouge, Parthes (wie Anm. 26), i 7.

46 Ov. ars I, I 78. Siehe dazu Lerouge, Parthes (wie Anm. 26), I I5-I I 9. Das Thema einer Eroberung des Partherreichs verschwindet mit Augustus.

47 Tonio Hölscher, Actium und Salamis, in: JbDAI 99, I984, I87-2 I4; Robert Alan Gurval, Actium and Augustus. The Politics and Emotions of Civil War. Ann Arbor 1995, 289; Cooley, Res Gestae (wie Anm.40), 2 Io, charakterisiert den Hintergrund der von Augustus inszenierten Naumachie folgendermaßen: „In this way, the mock naval battle evoked three different aspects of the west triumphing over the east under Augustus, namely Actium, recovery of the standards from Parthia, and Gaius' expedition.“

48 Jung, Marathon (wie Anm.30), 363 mit Anm. 88; Hardie, Images (wie Anm. 26), I 29. Freilich war der Ahnherr dieser Interpretation kein geringerer als Syme, Crisis (wie Anm.43), I5: „This piece of pageantry advertised Rome as the champion of Hellas against the Orient.“ 
Darüber hinaus wurden die technischen und inszenatorischen Aspekte dieser Naumachien, die sich nur aus vergleichenden Betrachtungen aller überlieferter Beispiele erschließen lassen, nicht gebührend beachtet. Kathleen M. Coleman hat deutlich gemacht, dass bei diesen Naumachien nicht von einem „Re-enactment“ im eigentlichen Sinn gesprochen werden kann. So ist allein schon aufgrund der Tatsache, dass es sich bei den beteiligten Personen um Kriegsgefangene und verurteilte Verbrecher handelt, die im wahrsten Sinne des Wortes um ihr Leben kämpften, zu schließen, dass es sich jeweils um ein Spektakel mit offenem Ausgang gehandelt hat. ${ }^{49}$ Denn nur so war auch ein entsprechender Spannungsbogen für das Publikum garantiert. Nicht zufällig waren die fiktiven Protagonisten dieser Spektakel niemals Römer - denn nur in diesem Fall wäre es wirklich problematisch gewesen, einen offenen Ausgang zuzulassen. ${ }^{50}$ Die Szenarien wurden vielmehr aus einer mythischen und pseudohistorischen Vergangenheit abgerufen, wobei Fragen der Authentizität gewiss eine untergeordnete Rolle spielten. ${ }^{5 \mathrm{I}}$ Mit Sicherheit sind beim allgemeinen Publikum auch keine tiefsitzenden Detailkenntnisse der verfügbaren Traditionen vorauszusetzen, sodass es letztlich unwesentlich war, wer hier als Sieger die Bühne verließ. Entscheidend waren vielmehr die für das stadtrömische Publikum exotischen und außergewöhnlichen Kontexte. ${ }^{52}$ Und dazu zählten sowohl das Sujet Seeschlacht als auch die Perserkriege. ${ }^{53}$

Welch geringe Bedeutung letztendlich dem Faktor Historizität zukam, zeigt sich bei der Inszenierung einer Naumachie, die Titus anlässlich der hunderttägigen Inaugurationsfeierlichkeiten bei der Eröffnung des Colosseums im stagnum Augusti zur Aufführung bringen ließ. Dabei traten Athener gegen Syrakusaner an, wobei der erfolglose Angriff Athens auf Syrakus im Jahre 4I4 v.Chr. das historische Kolorit darstellte. Entgegen der historischen Vorlage gewannen in diesem Fall die Athener, was aber keineswegs als Problem empfunden wurde. ${ }^{54}$ Nimmt man diesen Sachverhalt ernst, ist es wohl schwer vorstellbar, in der Aufführung der Seeschlacht von Sa-

\footnotetext{
49 Coleman, Launching into History (wie Anm. 39), 69, 72.

50 Ebd.72.

5I Ebd.69, 73f.

52 Ebd.70.

53 Ebd.73f.: „I believe that the evidence of the aquatic displays suggests that the classical age of Greece was sufficiently remote from imperial Rome for the actual and the mythical past to be accorded equal status in the popular imagination."

54 Ebd.48f., 67f., mit weiteren Beispielen für solche,Abweichungen` ebd.62f.
} 
lamis im stagnum Augusti eine vom Kaiser initiierte offizielle Botschaft zu sehen, die die Perserkriege im Sinne der Auseinandersetzung mit den Parthern ins allgemeine Bewusstsein rufen oder gar eine Gleichsetzung der Römer mit den Mitgliedern des Hellenenbunds propagieren sollte. Gebildete wie Ovid mögen hier Anklänge gesehen haben, zumal das inszenierte Ereignis ,zufällig' historisch akkurat ausging, doch sollte dies weder mit der Intention des Kaisers noch mit den Empfindungen eines Großteils des anwesenden Publikums verwechselt werden. ${ }^{55}$

Auch Nero soll eine Naumachie inszeniert haben. ${ }^{56}$ Die mittelbyzantinischen Exzerpte aus dem Geschichtswerk des Cassius Dio sprechen wohl darauf anspielend erneut von einer „Naumachie zwischen Persern und Athenern“57, die die moderne Forschung an den Beginn seines armenischen Krieges in das Jahr 57/58 n. Chr. datiert $^{58}$. Das Spektakel, das Caligula im Jahre 39 n.Chr. in der Bucht von Neapel organisierte, ist noch schwerer einzuordnen. ${ }^{59}$ So berichten Sueton und Cassius Dio ausführlich von einer Schiffsbrücke, die der junge Kaiser zwischen Baiae und Puteoli habe errichten lassen, über die er anschließend in prunkvoller Rüstung mit Truppen und Gefolge geschritten sei. ${ }^{60}$ Dabei habe er einen parthischen Knaben namens Dare(i)us mit sich geführt, der als „Geisel“ (obses) bei Caligula geweilt habe. Bemerkenswert ist allerdings, dass man schon zu Zeiten Suetons über den ideologischen Kontext der Inszenierung nicht mehr genau Bescheid wusste. ${ }^{6 \mathrm{I}}$ Jedenfalls wird der Leser mit drei verschiedenen Erklärungen konfrontiert, die angeblich in Umlauf (gewesen) seien. Caligula habe entweder Xerxes übertreffen oder aber die Germanen und Britannier mit einer jederzeit zu erwartenden römischen Invasion einschüchtern wollen. Schließlich wird noch eine in eine ganz andere Richtung zielende Erklärung

55 Paul Zanker, Augustus und die Macht der Bilder. München I987, 89, und Gurval, Actium (wie Anm.47), 289, sahen in der Naumachie eine Reminiszenz auf Actium und den Sieg des Westens über den barbarischen Osten. Aber auch diese Botschaft lässt sich schwer durch ein Spektakel transportieren, dessen Ausgang offen war. Vgl. auch Hardie, Images (wie Anm. 26), I39f.

56 Suet. Nero I 2; Coleman, Launching into History (wie Anm. 39), 56f., 69, die wiederum betont, dass der Ausgang wohl offen war.

57 Cass. Dio 6r b,9,5.

58 Spawforth, Unity (wie Anm. 26), 238.

59 Vgl. S. J. V. Malloch, Gaius' Bridge at Baiae and Alexander-imitatio, in: CQ 5I, 200I, 206-2 I 7, der deutlich macht, wie der Tyrannendiskurs der erhaltenen Quellen die eigentlichen Motive des Kaisers für das Spektakel verdunkelt.

60 Suet. Cal. I9; Cass. Dio 59, I 7. Weitere Quellen: Ios. Ant. Iud. I9,6; Sen. brev. I8,5-6.

6I Möglich ist freilich auch, dass der Tyrannendiskurs den ursprünglichen Kontext bewusst zudeckte. 
geboten: Caligula habe den Hofastrologen des Tiberius, Thrasyllos, Lügen strafen wollen, habe dieser doch einst seinem Vorgänger prophezeit, „Gaius werde genau so wenig Kaiser werden, wie er die Bucht von Baiae zu Pferd überqueren könne“ ${ }^{62}$

Auch bei Cassius Dio wird ein bunter Mix an Motiven geboten, die den Kaiser zu diesem Spektakel veranlasst hätten. ${ }^{63}$ Wie bei Sueton bleibt offen, inwieweit hier Authentisch-Zeitgenössisches mit späteren Deutungen vermischt und in einen Topf geworfen wurde. So zieht Caligula hier als Alexander hoch zu Ross, begleitet von seinen Truppen wie auf einem Feldzug von Baiae nach Puteoli, wo er eine imaginäre Schlacht schlägt. Die Rückkehr erfolgt als Triumphator auf einem Streitwa-

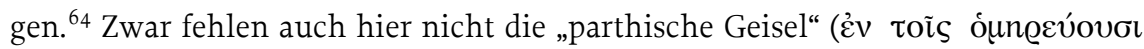

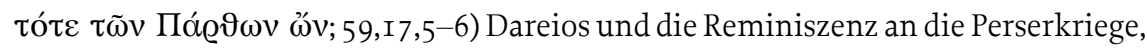
doch taucht neben dem Alexander-Motiv auch eine allgemeine Übertreffungsmetaphorik auf. ${ }^{65}$ Diese wird keineswegs nur gegenüber den Perserkönigen ausgebreitet $^{66}$, sondern sogar gegenüber der Gottheit ins Spiel gebracht. Als Caligula nämlich in seinem Überschwang einen Teil der ihn begleitenden Gefährten von der Brücke stößt und diese wider Erwarten nicht ertrinken, schreibt er auch dies seiner besonderen Stellung zu: „Den Kaiser erfüllte aber auch dieser Umstand nicht wenig mit Stolz; denn er erklärte, selbst Poseidon habe vor ihm Angst bekommen. “67 Dahinter

62 Suet. Cal. I9,3.

63 Siehe zu Cassius Dio jetzt Carsten Hjort Lange/Jesper Majbom Madsen (Eds.), Cassius Dio. Greek Intellectual and Roman Politician. Leiden/Boston 2016.

64 Nach Malloch, Gaius' Bridge (wie Anm. 59), 2 I I f., habe Caligula keinen Triumph im eigentlichen Sinn im Auge gehabt, sondern den bei Curt. 9,I0,27 beschriebenen bacchischen Umzug Alexanders in Karamanien nachgeahmt. Carsten Hjort Lange, Mock the Triumph. Cassius Dio, Triumph and Triumph-Like Celebrations, in: ders./Majbom Madsen (Eds.), Cassius Dio (wie Anm.63), ro6f., spricht dem Geschehen hingegen durchaus den Charakter eines Triumphes zu.

65 Zum Motiv der Übertreffungsmetaphorik als eine für Imperien genuine Ausdrucksform eines, Weltherrschaftsanspruchs' siehe Michael Gehler/Robert Rollinger, Imperien und Reiche in der Weltgeschichte. Epochenübergreifende und globalhistorische Vergleiche, in: dies. (Hrsg.), Imperien in der Weltgeschichte. Epochenübergreifende und globalhistorische Vergleiche. 2 Bde. Wiesbaden 20I4, Bd. I, I-32. Vgl. auch Coleman, Launching into History (wie Anm. 39), 66, 68. Auch Malloch, Gaius' Bridge (wie Anm. 59), 208, 2 I 4 f., sah diesen Aspekt, doch habe Caligula im konkreten Fall Alexander nicht übertreffen (aemulatio), sondern sich als neuer Alexander stilisieren wollen (imitatio).

66 Cass. Dio 59,I 7, I I: „Was aber Darius und Xerxes betraf, so goss er die ganze Schale seines Spottes über sie aus und behauptete von sich, dass er eine vielfach größere Meeresstrecke als sie überbrückt habe“; dt. Übersetzung nach: Cassius Dio, Römische Geschichte (wie Anm.42), Bd. 4, 400.

67 Cass. Dio 59,I 7, I I; dt. Übersetzung nach Cassius Dio, Römische Geschichte (wie Anm. 42), Bd. 4, 399 f. 
lässt sich der Anspruch erkennen, selbst die Elemente zu bezwingen ${ }^{68}$, was auch expressis verbis zweimal festgehalten wird ${ }^{69}$. Das Compositum Mixtum an Motiven mahnt zur Vorsicht, die Perserkriege als das für den römischen Kaiser authentische Ideologem herauszustreichen oder gar jeweils unterschiedliche Inszenierungen zu rekonstruieren, denen dann ein spezifischer Deutungshorizont zugewiesen wird. ${ }^{70}$

Aus all dem lässt sich vielmehr die Vielfalt der Deutungs- und Erklärungshorizonte erschließen, die die genannten römischen Kaiser bei ihren Inszenierungen im Kopf gehabt haben mögen. Dabei ist freilich zusätzlich mit einer breiten Rezeptionsebene zu rechnen, wobei sich jeweils neue Interpretationsspielräume erschlossen haben mögen. Die Perserkriege haben dabei sicherlich eine gewisse Rolle gespielt, doch scheint diese nicht besonders dominant gewesen zu sein. ${ }^{7 \mathrm{I}}$ Dies ist bei näherer Betrachtung durchaus naheliegend, hatte der historische Vergleich mit den Perserkriegen doch nicht nur deutliche Schwachstellen, sondern barg womöglich auch ein gewisses Unruhepotential.

68 Am besten fassen lässt sich dieser Anspruch bei den achaimenidischen Königen, wie er etwa in der Tat bei der Schiffsbrücke des Xerxes, bei dessen Durchstich durch die Athos-Halbinsel oder etwa bei Dareios' Kanalbau zwischen Nil und Rotem Meer zum Ausdruck kommt: Benedikt S. J. Isserlin, The Canal of Xerxes, in: The Annual of the British School at Athens 86, I991, 83-9I; Robert Rollinger, Das teispidisch-achaimenidische Großreich. Ein ,Imperium‘ avant la lettre?, in: Gehler/Rollinger (Hrsg.), Imperien in der Weltgeschichte (wie Anm.65), Bd. I, I49-I92; ders., Monarchische Herrschaft am Beispiel des teispidisch-achaimenidischen Großreichs, in: Stefan Rebenich/Johannes Wienand (Hrsg.), Monarchische Herrschaft im Altertum. Berlin 2017, I89-2 I5. Die griechische Tradition interpretierte dies als Hybris und unterdrückte demgegenüber den in diesen Leistungen inhärenten Könnensstolz, der bewusst die Beherrschung der Natur inszeniert. Letzteres zu demonstrieren war aber keinesfalls ein ausschließlich ,orientalischer‘ Habitus, sondern ließ auch römische Kaiser nicht unberührt; vgl. Coleman, Launching into History (wie Anm.39), 63, 66f., 69, 74. Stellt man dies in Rechnung, könnte man Caligulas Inszenierung durchaus auch als eine aemulatio Xerxis verstehen; vgl. Coleman, Launching into History (wie Anm.39), 69; Hardie, Images (wie Anm. 26), I32.

69 Cass. Dio 59,I7,I: „Hingegen verlangte ihn sehr, irgendwie mit Pferden über das Meer zu fahren“; dt. Übersetzung nach: Cassius Dio, Römische Geschichte (wie Anm.42), Bd. 4, 398. Cass. Dio 59,I 7,7: „Und zuerst rühmte er sich selbst als den Veranlasser gewaltiger Unternehmen, dann pries er die Soldaten als Männer, die sich Mühen und Gefahren unterzogen hätten, und erwähnte insbesonders, dass sie zu Fuß über das Meer marschiert seien“; ebd. 399.

70 Lerouge, Parthes (wie Anm. 26), I48. Bezeichnenderweise referiert Lerouge nur zwei der drei von Sueton gebotenen Erklärungsmodelle; so auch Hardie, Images (wie Anm. 26); Makhlaiuk, Memory (wie Anm. 26). Will man unbedingt versuchen, ein authentisches Handlungsmotiv zu rekonstruieren, so bietet sich der mit der bereits angesprochenen Übertreffungsmetaphorik verbundene Weltherrschaftsanspruch an. 7 I Lerouge, Parthes (wie Anm. 26), Hardie, Images (wie Anm. 26), und Makhlaiuk, Memory (wie Anm. 26) sehen dies anders. 
So repräsentierte der aus etwa dreißig griechischen Poleis bestehende Hellenenbund, der sich den Persern unter spartanischem Kommando entgegenstellte, keineswegs die Gesamtheit der mehrere hundert Poleis umfassenden griechischen Gemeinwesen. Jedenfalls fochten deutlich mehr griechische Poleis auf der Seite des Großkönigs als auf jener Athens und Spartas. Auch der Umstand, dass der Hellenenbund nach dem Rückzug Spartas an Bedeutung verlor und sich der neu gegründete Attische Seebund zusehends zu einem Vehikel des athenischen Imperialismus entwickelte, in den die neue Seemacht Athen Bündner auch durch Gewalt und Zwang presste $^{72}$, mag nicht unbedingt allenthalben zu einer positiven Rezeption der Perserkriege in der griechischen Welt beigetragen haben. Darüber hinaus waren seit hellenistischer Zeit völlig neue politische Landschaften entstanden, die sich nur schwer mit den alten Deutungshorizonten und Geschichtsbildern verbinden ließen. Griechisch-hellenische Identitäten machten sich in diesem Zusammenhang auch in Vorderasien breit, wobei dies oft nur ein vergleichsweise oberflächliches Konstrukt darstellte. Dabei war die Polisidentität wohl stets zentraler als die Zugehörigkeit zu den Hellēnes. Der Polis kam als patris stets die entscheidende Bedeutung zu. Dies lässt sich über die ganze hellenistische Zeit hinweg beobachten und änderte sich auch nicht, als seit dem I.Jahrhundert v.Chr. immer größere Teile Vorderasiens unter römische Oberhoheit gerieten und provinzialisiert wurden. Der Ost-West-Gegensatz zwischen Rom und den Parthern, wenn er denn als solcher empfunden wurde, wurde somit in völlig neuen geographischen Räumen ausgefochten, deren historische Erinnerungshorizonte sehr heterogen waren. So hatte natürlich auch das persischachaimenidische Imperium in diesen Regionen seine Spuren hinterlassen, die mit entsprechenden, durchaus heterogenen Geschichtsbildern verbunden waren. Dazu gehört nicht nur das von Antony Spawforth angeführte Beispiel einer Dame senatorischen Standes aus Selge, die den Namen Aurelia Volussia Quirina Atossa trug und gemeinsam mit ihrem Ehemann Magnianus Perikles als Kaiserpriesterin und Kaiserpriester agierte. ${ }^{73}$ Auch sonst hat die neuere Forschung auf die starke Präsenz persischer Traditionen etwa im römischen Kleinasien aufmerksam gemacht, wo ent-

72 Kai Ruffing, Reparationen, Tribut, Enteignung. Wirtschaftliche Folgen des Krieges am Beispiel des Delisch-Attischen Seebundes, in: Lena Meier/Oliver Stoll (Hrsg.), Niederlassung und Kriegsfolgen - Vae Victis oder Vae Victoribus? Vom Alten Orient bis ins Europäische Mittelalter. Berlin 20I6, 29-45.

73 Spawforth, Unity (wie Anm. 26), 247, mit Verweis auf Johannes Nollé/Friedel Schindler, Die Inschriften von Selge. (Inschriften griechischer Städte aus Kleinasien, Bd. 37.) Bonn I99I, 95-97, Nr. 20. 
sprechende Kulte und Priesterämter bis weit in die römische Kaiserzeit hinein gut bezeugt sind. ${ }^{74}$

All dies offenbart das vergleichsweise geringe Mobilisierungspotential der Perserkriege in der römischen Kaiserzeit. In Athen und Sparta sowie den wenigen Poleis, die sich beim Hellenenbund engagiert hatten, mag dies anders gewesen sein ${ }^{75}$, doch für die überwiegende Mehrzahl der griechischsprachigen Gemeinwesen im östli-

74 Stephen Mitchell, Iranian Names and the Presence of Persians in the Religious Sanctuaries of Asia Minor, in: Elaine Matthews (Ed.), Old and New Worlds in Greek Onomastics. (Proceedings of the British Academy, I48.) London 2007, I5 I-I 7 I; Bruno Jacobs, Tradition oder Fiktion? Die, persischen' Elemente in den Ausstattungsprogrammen Antiochos' I. von Kommagene, in: Strootman/Versluys (Eds.), Persianism in Antiquity (wie Anm. 26), 235-248; Charlotte Lerouge-Cohen, Persianism in the Kingdom of Pontic Kappadokia. The Genealogical Claims of the Mithridatids, in: ebd.223-233; Valeria Sergueenkova/Felipe Rojas, Persia on Their Minds: Achaemenid Memory Horizons in Roman Anatolia, in: ebd. 269-288; vgl. auch Robert Rollinger, Altorientalisches bei Herodot. Das wiehernde Pferd des Dareios I., in: Hilmar Klinkott/ Norbert Kramer (Hrsg.), Zwischen Assur und Athen. Altorientalisches in den Historien Herodots. (SpielRäume der Antike, 4.) Stuttgart 20I7, 4I f. In diesen Kontext gehört auch der Umstand, dass sich zahlreiche griechische Städte Kleinasiens als Gründungen prominenter Amazonen verstanden; Hardie, Images (wie Anm. 26), I38 Anm. 35. Dieses Selbstverständnis ist deshalb bemerkenswert, weil die Amazonomachie als Sinnbild für den Perserkrieg galt.

75 Spawforth, Unity (wie Anm. 26), 246, spricht dabei pointiert von „Athenian self-congratulation“. So konnte etwa Athen im I.Jahrhundert n. Chr. durch die großzügige Unterstützung des Julius Nikanor aus Hierapolis die Insel Salamis erwerben, die seit Sulla nicht mehr im Besitz der Stadt war. Der großzügige Wohltäter wurde prompt als „,neuer Themistokles“vorgeführt; Spawforth, Unity (wie Anm. 26), 243 f.; Christopher P. Jones, Three Foreigners in Athica, in: Phoenix 32, 1978, 222-228; T. Leslie Shear, Athens. From CityState to Provincial Town, in: Hesperia 50, I98I, 366-367. Dio Chrysostomos übt allerdings Kritik an dem Nikanor zugesprochenen Ehrentitel, was wohl nicht den nostalgischen und überhöhten Blick auf die eigene Vergangenheit betraf, sondern eher die Unverhältnismäßigkeit des Ehrentitels ansprach (Dion Chrys. 3I,I I6). Jedenfalls zeigen mehrere der Nikanor zugeeigneten Inschriften Spuren einer Rasur, die den Ehrentitel zu beseitigen trachteten; vgl. Jones, Foreigners (wie Anm.75), 222 mit Anm. I, der ebd. 223 Anm. 5

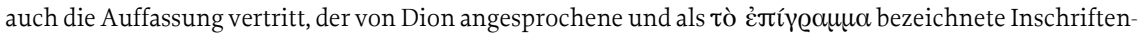
text auf der Statue müsse ein Epigramm im eigentlichen und nicht eine Inschrift im herkömmlichen Sinne bezeichnet haben. In Megara ist ein jährliches Stieropfer zu Ehren der in verschiedenen Schlachten der Perserkriege gefallenen Polisbürger bezeugt (genannt werden Kap Artemision, Mykale, Salamis und die „Böotische Ebene“ = Platäa), das wohl unmittelbar nach dem Abzug der Perser eingerichtet wurde. Die auf dieses Ereignis bezugnehmende Inschrift ist allerdings eine,Wiederaufzeichnung، aus der Zeit des 4.-5.Jahrhunderts n. Chr.: Charles W. Fornara, Archaic Times to the End of the Peloponnesian War. (Translated Documents of Greece and Rome, Vol. I.) $2^{\text {nd }}$ Ed. London/New York/New Rochelle I983, 58f., Nr.60 = Kai Brodersen/Wolfgang Günther/Hatto H.Schmitt (Hrsg.), Historische Griechische Inschriften in Übersetzung. Bd. I: Die Archaische und Klassische Zeit. (Texte zur Forschung, 59.) Darmstadt I 992, 25, Nr.43; vgl. Spawforth, Unity (wie Anm. 26), 243. Zur Erinnerungskultur des römischen Sparta mit seinen Monumenten, Gräbern und Festen siehe Paul Cartledge/Antony Spawforth, Hellenistic and Roman Sparta: A Tale of Two Cities. London/New York I989, I90-I93. 
chen Teil des Imperium Romanum hatten die Perserkriege wohl nur geringe Bedeutung für die unmittelbare Gegenwart, zumindest was deren politische Instrumentalisierung betraf. Das von Kaiser Hadrian ins Leben gerufene Panhellenion bietet hierfür ein willkommenes Anschauungsbeispiel. ${ }^{76}$ Es verdankte seine Gründung einer römisch-imperialen Initiative, die den Kaiserkult mit einer auf Athen und Eleusis fokussierten Einrichtung verband, die das ,alte Griechenland repräsentieren sollte. ${ }^{77}$ Dabei kam eine spezifisch römische Sicht dessen zum Ausdruck, was als ,genuin“ griechisch zu gelten habe und was nicht. Aus diesem Grund dürfte die Mitgliedschaft tatsächlich auf „rechtgeborene“ (iછaycveĩs) Griechen beschränkt gewesen sein, die man sich als Abkömmlinge dorischen, ionischen oder äolischen Blutes imaginierte. ${ }^{78}$ Schließlich versammelte der Bund auch nur Griechen aus den römischen Provinzen Achaea, Macedonia, Thracia, Asia sowie Cyprus et Cyrene. Griechen aus dem Westen waren wohl ebenso ausgeschlossen wie jene der römischen Ostprovinzen. ${ }^{79}$

Aber auch in den als authentisch geltenden Kernprovinzen hielt sich die Zustimmung offenbar in Grenzen. Dies war wohl nicht nur einem gewissen Unmut bezüglich der Definition des Hellenischen geschuldet, sondern betraf auch den Umstand, dass die Mitgliedschaft im Panhellenion in gewisser Weise eine Akzeptanz des Primats Athens miteinschloss, das als Zentrum des Bundes fungierte. Das war für selbstbewusste Poleis wie Ephesos, Pergamon und Smyrna eine unzumutbare Hürde, weshalb sie sich auch nicht am Panhellenion beteiligten. ${ }^{80}$ So offenbart die Geschichte des Panhellenions, das bald nach dem Ableben Hadrians erste Krisensymptome zeigte ${ }^{8 \mathrm{I}}$, eindrucksvoll die Probleme, die mit einem Rekurs auf eine vermeint-

76 Antony Spawforth, s. v. Panhellenion, in: Der Neue Pauly. Bd. 9. München 2000, 247. Christopher P. Jones, The Panhellenion, in: Chiron 26, I996, 29-56, sieht die Initiative zur Gründung nicht beim Kaiser, sondern bei den griechischen Poleis.

77 Antony Spawforth, The Panhellenion Again, in: Chiron 29, I999, 344-47; Kaja Harter-Uibopuu, Kaiserkult und Kaiserverehrung in den Koina des griechischen Mutterlandes, in: Hubert Cancik/Konrad Hitzl (Hrsg.), Die Praxis der Herrscherverehrung in Rom und seinen Provinzen. Tübingen 2003, 209-23I.

78 Jones, Panhellenion (wie Anm. 76), 47-54, und Spawforth, Panhellenion Again (wie Anm. 77), 349f., mit Verweis auf einen inschriftlich erhaltenen Brief Hadrians an den Archon des Panhellenion, der die Frage einer möglichen Mitgliedschaft von Ptolemais-Barka thematisierte.

79 Spawforth, Panhellenion Again (wie Anm.77), 347-352. Gegen die Evidenz postuliert Jung, Marathon (wie Anm.30), 370, hingegen, dass sich unter dem Dach des Panhellenion „Vertreter der gesamten griechischsprachigen Welt" zusammengefunden hätten.

80 Spawforth, Panhellenion Again (wie Anm.77), 343.

8I Ebd.35of. 
lich gemeinsame griechische Vergangenheit verbunden waren. Hier war im Sinne einer Auseinandersetzung des Imperiums mit den Parthern nur mit einer beschränkten Mobilisierungskraft zu rechnen. ${ }^{82}$ Dies musste umso mehr gelten, wenn diesem Rekurs keine religiöse, sondern eine politische Botschaft innewohnte, wie das bei dem Bezug auf die Perserkriege der Fall gewesen sein soll. Eine gemeinsame griechisch sprechende Öffentlichkeit war damit ebenso wenig zu instrumentalisieren, wie die römische Öffentlichkeit wohl ein spezifisches und, in den Augen einer griechischsprachigen Bevölkerung, wohl zum Teil auch eigentümliches Bild von dem hatte, was als "griechisch“ zu gelten hatte. ${ }^{83}$

Freilich mag die einseitige Fokussierung einzelner römischen Kaiser - wie auch der modernen Forschung - auf Athen den Blick auf das „Hellenische“ etwas verzerrt haben. In diesem Fall sollten entsprechende Äußerungen, wie sie etwa in der griechisch-römischen Historiographie auftauchen, nicht sofort als Teil einer ,offiziellen` Reichsideologie verstanden werden. ${ }^{84}$ Diese scheint vielmehr schon seit den Tagen des Pompeius sehr stark um eine Alexander-Imitatio bemüht gewesen zu sein, die mit einem universellen Weltherrschaftsanspruch verbunden war, wie er auch in authentischen Zeugnissen, wie Augustus' Res Gestae oder dem Programm des Augustus-Forums zum Ausdruck kam. ${ }^{85}$ Dass man in diesem Zusammenhang auch immer wieder auf die Perserkriege angespielt haben mag, verwundert nicht, waren diese

82 Was Spawforth (ebd.35I) abschließend zur Wirkkraft des Panhellenion festhält, mag deshalb grosso modo auch für die Bedeutung der Perserkriege in dieser Zeit gelten: „The interest of the Panhellenion now seems to lie less in its impact on the Greek-speaking provinces, which may have been relatively restrained, especially beyond the mid-second century, than in what it reveals about the thinking of its founder (scil. Hadrian).“

83 Ebd.352: „a particular (Roman) construction of Greekness“.

84 Man vgl. in diesem Zusammenhang Ernst Baltrusch, Historia als Waffe. Zu den politischen Möglichkeiten der griechischen Geschichtsschreibung im Imperium Romanum, in: Hans Beck/Benedikt Eckhardt/ Christoph Michels/Sonja Richter (Hrsg.), Von Magna Graecia nach Asia Minor. Festschrift für Linda-Marie Günther zum 65. Geburtstag. (Philippika. Altertumswissenschaftliche Abhandlungen, I r6.) Wiesbaden 2017, 267-279, der einzelne Beispiele der griechischsprachigen Historiographie des Imperium Romanum als Stimme der ,Ohnmächtigen“ versteht, die sich „,in einer übermächtigen Umwelt einen festen Standpunkt zu verschaffen (suchen)“.

85 Lerouge, Parthes (wie Anm. 26), I 23 f., I46-I 49; vgl. auch Christof Schuler, Augustus, Gott und Herr über Land und Meer. Eine neue Inschrift aus Tyberissos im Kontext der späthellenistischen Herrscherverehrung, in: Chiron 37, 2007, 383-403; Claude Nicolet, Space, Geography and Politics in Early Roman Empire. Michigan I99I (Ndr. 20I5); Almagor, Empire (wie Anm. 26), 34I mit Anm.73 und 74, betont die altorientalischen Wurzeln dieses Ideologems. 
doch ein fester Bestandteil der von den Eliten gepflegten Bildungstradition. ${ }^{86}$ Diese findet nicht nur ihren Niederschlag in den uns erhaltenen historiographischen Werken ${ }^{87}$, sondern machte sich besonders nachhaltig bei den Gelehrten der Zweiten Sophistik bemerkbar, die einen nicht unbeträchtlichen Teil ihres Themenreservoirs aus dieser Tradition schöpften ${ }^{88}$. Dabei dürfte allerdings kaum eine zeitgenös-

86 Siehe dazu etwa Donald Andrew Russell, Greek Declamation. Cambridge/London/New York I983, I06-I28; Bridges, Xerxes (wie Anm. 26), I 27-I89.

87 Bemerkenswert ist freilich auch, dass man von parthischer Seite im I.Jahrhundert n. Chr., folgt man der klassischen Überlieferung, den römischen Weltherrschaftsanspruch akzeptiert zu haben scheint oder die Römer zumindest glauben ließ, dass dem so sei. Dies hat Lerouge, Parthes (wie Anm. 26), I46-I49, überzeugend herausgearbeitet, wobei sie ebenso klarmacht, dass dahinter einerseits eine Strategie stand, die bei einem tatsächlichen Gleichgewicht der Kräfte - einfach römische Einfälle ins Partherreich verhindern wollte, andererseits die damit einhergehende ,Botmäßigkeit‘ explizit nur von den parthischen Gesandten in Rom sowie dort weilenden parthischen,Geiseln' vertreten wurde, die hofften, mit Roms Hilfe auf den parthischen Thron zu gelangen. Auch dies sind freilich keine authentischen Zeugnisse jener Herrschaftsideologie, wie sie von regierenden parthischen Königen vertreten wurde. Diese wird vielmehr dann fassbar, wenn es wirklich um etwas ging, was etwa immer dann der Fall war, wenn die Herrschaft über Armenien auf dem Spiel stand. Dann war nämlich Schluss mit dem parthischen (Pseudo-)Klientelstatus. Trotzdem ist das Verhalten der parthischen Gesandten und ,Geiseln“ in Rom auch in einer weiteren Perspektive interessant. So legen zumindest die uns erhaltenen Quellen nahe, dass der im alten Vorderasien zum ersten Mal seinen Ausgangspunkt nehmende universelle Weltherrschaftsanspruch imperialer Staatsgebilde im Falle Roms einen viel stärkeren Niederschlag gefunden hat, als wir das etwa für das Partherreich erschließen können; siehe dazu auch Christoph Michels, Reich und Reichsgedanke auf den Münzen der Antoninen. Überlegungen zu Hadrians und Pius' Provinzserien, in: Benedikt Eckhardt/Katharina Martin (Hrsg.), Eine neue Prägung. Innovationspotentiale von Münzen in der griechisch-römischen Antike. Wiesbaden 20 16, 69-7 I. Vgl.zu diesem universellen Weltherrschaftsanspruch und dessen altorientalischem Hintergrund Rollinger, Großreich (wie Anm.76), bzw. zu den parthischen und römischen ,Pendants‘ Kai Ruffing, Rom - das paradigmatische Imperium, in: Gehler/Rollinger (Hrsg.), Imperien in der Weltgeschichte (wie Anm.65), Bd. I, 40I-447; Josef Wiesehöfer, Parther und Sasaniden. Imperien zwischen Rom und China, in: ebd.449-478; $M$. Rahim Shayegan, Arsacids and Sasanians. Political Ideology in Post-Hellenistic and Late Antique Persia. Cambridge 20II, 39-33I; ders., Persianism. Or Achaemenid Reminiscences in the Iranian and Iranicate World(s) of Antiquity, in: Strootman/Versluys (Eds.), Persianism in Antiquity (wie Anm. 26), 40I-455. Auch das im alten Vorderasien gut bezeugte Postulat der ,ewigen Herrschaft', das mit der Idee des für das Wohlergehen seines Volkes sorgenden Potentaten aufs engste verknüpft war, lässt sich übrigens gerade für die römische Kaiserzeit gut nachweisen: Peter F. Mittag, Die Ewigkeit des Kaisers und das Wohlergehen des Reiches, in: Beck u.a. (Hrsg.), Von Magna Graecia nach Asia Minor (wie Anm. 84), 339-347.

88 Russell, Declamation (wie Anm. 86), I07, der präzisiert: „In all, we know of about 350 themes of Greek history treated by the declaimers. A few are mythological, 32 deal with the Persian war, about 90 with the Peloponnesian war, 25 with the period of Demosthenes, and 25 or so with Alexander." Zieht man ins Kalkül, dass Demosthenes eigentlich als ,Perserfreund‘ galt (Almagor, Empire [wie Anm. 26], 329f.), so relativiert sich die Bedeutung der Perserkriege, was ihre ideologische Instrumentalisierungskraft betrifft, auch in diesem Kontext. 
sische politische Instrumentalisierung eine Rolle gespielt haben. ${ }^{89}$ Vielmehr dienten solche Reminiszenzen einer gelehrten Demonstration des Wissens, die auch mit Amüsement verbunden sein konnte. ${ }^{90}$ Dies zeigt etwa eindrucksvoll Philostrat unter Verweis auf Skopelianos von Klazomenai ${ }^{9 \mathrm{I}}$, der sich offensichtlich trefflich darauf verstand, die Perserkönige Dareios und Xerxes wild gestikulierend und in angetrunkenem Zustand einem begeisterten Publikum vorzuführen:

„Vortrefflich verstand er sich auf figurenreiche Reden und uneindeutige Ausdrücke; noch bewunderungswürdiger aber war er in der Behandlung von Themen, die mehr Affekt erforderten, und am meisten in den Gegenständen aus der medischen (persischen) Geschichte, etwa in seinem ,Dareios“ und seinem ,Xerxes'; diese scheint er mir nämlich am besten unter allen Sophisten dargestellt und damit den Späteren ein Muster der Darstellung gegeben zu haben: Er drückte den Übermut und die Schlichtheit im Charakter der Barbaren aus und soll dabei auch in lebhafter Bewegung gewesen sein, wie ein von (dem Weingott Bacchus) Begeisterter. Als daher einer von den Schülern des Polemon sagte, er schlage die Pauke, griff Skopelianos diesen Scherz auf und erwiderte: ,Ich schlage - aber den Schild des Aias! “ “92

Schließlich waren die Perserkriege im griechischen Bewusstsein fest als Freiheitskriege verankert, weshalb in Platäa auch ein Tempel des Zeus Eleutherios stand. Die-

89 Laut Gerhard Jean Daniël Aalders/Lukas de Blois, Plutarch und die politische Philosophie der Griechen, in: Aufstieg und Niedergang der Römischen Welt. T.2, Bd. 36.5. Berlin/New York I992, 3402 f., dürfte „die übermäßige Beschäftigung mit der klassischen Vergangenheit in diesen Kreisen (scil. der Sophisten) eine Kompensation für das Fehlen wirklicher politischer und militärischer Gewalt geboten haben. Russell, Declamation (wie Anm. 86), I09, sah dies ähnlich: „In general, the concentration on the past must have been escapism.“ Almagor, Empire (wie Anm. 26), 328, bezeichnet die Haltung der Sophisten als „nostalgic“ und erkennt eine „artificially simplistic world view“.

90 Almagor, Empire (wie Anm. 26), $333 \mathrm{f}$.

9I Vgl.zu Philostrat Graeme Miles, Philostratus. Interpreters and Interpretation. London/ New York 2018; Almagor, Empire (wie Anm. 26), 332, betont den Unterhaltungswert solcher Präsentationen; Miles, Philostratus (wie Anm.9I), 38, unterstreicht ,the ideal of an active interpreter, whose innate, mimetic capacity recreates the objects of interpretation, with constant reference to inherited paideia“. Dabei musste natürlich die Erwartungshaltung des Publikums befriedigt werden.

92 Philostr. soph. I,2 I, 5. Deutsche Übersetzung nach: Philostratos, Leben der Sophisten. Hrsg. v. Kai Brodersen. Wiesbaden 20I4, 79. Zum Topos der angeblich besonders ausgeprägten persischen Trunksucht in der klassischen Überlieferung vgl. Barbara Mauritsch-Bein, Trinkverhalten bei Fremdvölkern aus der Sicht antiker Autoren - dargestellt am Beispiel der Perser, Thraker und Skythen, in: Robert Rollinger/Christoph Ulf (Hrsg.), Geschlechter - Frauen - Fremde Ethnien in antiker Ethnographie, Theorie und Realität. Innsbruck/Wien/München 2002, 79-II8. 
se Freiheit war seit dem Ende des 4.Jahrhunderts v.Chr. zusehends eine Chimäre, wobei die griechischen Poleis am Ende gänzlich unter römische Oberhoheit gerieten. Die Perserkriege mögen einige griechische Poleis daran erinnert haben, dass sie letztendlich nicht Teil des „Imperiums des Ostens“ geworden waren, doch machte das Erinnern an sie wohl ebenso offensichtlich, dass sie nun ein Teil des „Imperiums des Westens“ waren. ${ }^{93}$ Der Idee der Freiheit haftete hier deshalb sehr wohl etwas Umstürzlerisches an.

Vor diesem Hintergrund überrascht es nicht, dass das ständige Wiederholen der angeblichen Bedeutung der Perserkriege in den kaiserzeitlichen Gegenwarten seit dem ausgehenden I.Jahrhundert n.Chr. auch von griechischsprachigen Intellektuellen kritisch betrachtet werden konnte. Dazu sind uns zumindest drei wichtige Stimmen erhalten. ${ }^{94}$ Dabei störte Flavius Josephus in seiner Einleitung zum Jüdischen Krieg weniger das mögliche Unruhepotential des Narrativs als vielmehr das vermeintlich nicht enden wollende Aufkochen der immer gleichen Geschichten, denen nicht nur jeglicher Neuigkeitswert mangele, sondern - und dies ist für unseren Zusammenhang noch wichtiger - denen im Vergleich zu den welthistorischen Ereignissen der Gegenwart nicht die Bedeutung zukomme, die ihnen gemeinhin zugeschrieben werde. ${ }^{95}$ Bei Josephus ist mit diesen epochalen Ereignissen der unmittelbaren Gegenwart natürlich der Jüdische Krieg gemeint. ${ }^{96}$

Eine ähnliche Tonlage schlägt Lukian an, der mit scharfer Zunge den in sophistischen Kontexten inflationären Gebrauch der Perserkriegs-Topik auf die Schippe nimmt ${ }^{97}$ :

„Gesetzt Du hättest zu Athen von irgendeinem Räuber oder Ehebrecher zu sprechen, so sprich du von dem, was in Indien und zu Ekbatana geschieht: vor

93 So war das Panhellenion auch ein Instrument des Kaiserkults, wobei in einem eigens in Athen oder Eleusis errichteten Tempel zwar nicht dem Xerxes, doch immerhin dem Hadrian Panhellenius und seinen Nachfolgern geopfert wurde; Spawforth, Panhellenion Again (wie Anm. 77), 344-347.

94 Auf zwei (Flavius Josephus, Plutarch) machte bereits Spawforth, Unity (wie Anm. 26), 244f., aufmerksam.

95 Vgl. zu Flavius Josephus und dem Kontext seiner Werke Ernst Baltrusch, Flavius und Josephus - zwei Seelen in einer Brust?, in: Richard Faber/Achim Lichtenberger (Hrsg.), Ein pluriverses Universum. Zivilisationen und Religionen im antiken Mittelmeerraum. Paderborn 2015, 369-390.

96 Ios. Bell. Iud. I,5, I3. Siehe dazu Baltrusch, Historia (wie Anm. 84), 275-278, der dem Werk des Josephus ein „antigriechisches Wettbewerbskonzept“ bescheinigt.

97 Lukian, Rhetorum Praeceptor I 8; Almagor, Empire (wie Anm. 26), 333: „[...] these typical historical instances from the Persian Wars were estimated an essential constituent of any effective speech“. 
allem anderen aber vergiss mir das Treffen bei Marathon und den Cynaegeirus nicht, ohne welche gar nichts zu machen ist; auch muss immer um den Athos herumgeschifft und zu Fuß über den Hellespont gegangen werden, Xerxes fliehen, Leonidas der Held des Tages sein, der Brief, den Othryades mit seinem Blute schrieb, vorgelesen und Salamis, Artemisium und Platäa tapfer herausgestrichen werden! Je dichter das alles aufeinander gedrängt wird, desto besser!“ 98

Noch bemerkenswert ist vielleicht die Kritik Plutarchs, macht er doch mehr als deutlich, dass der Bezug auf die Perserkriege eigentlich eine Sache Athens - und damit keineswegs der gesamten griechischsprachigen Welt - sei. Das Erinnern an diese angeblichen Großtaten der Vergangenheit sei zunehmend lächerlich und deplatziert, gebe es in der athenischen Geschichte doch genug Ereignisse, auf die die Athener auch jetzt noch stolz sein könnten und auf die sie sich doch eher berufen sollten. Dabei kommt er auch deutlich auf die Gefahren zu sprechen, die die Benutzung des Perserkrieg-Narrativs mit sich bringe, „rege es doch die Massen in lächerlicher Art

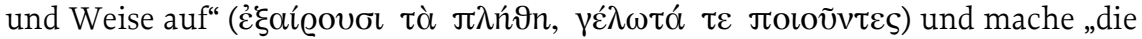

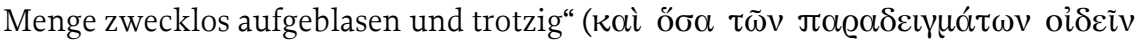

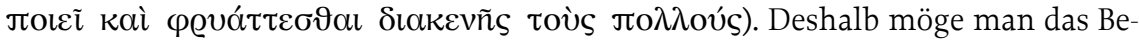
mühen dieses Narrativs besser den Sophisten überlassen. ${ }^{99}$ Plutarch bestätigt damit, dass das offensichtlich vielzitierte Narrativ der Perserkriege in sophistischem Kontext keine politische Sprengkraft besaß, sondern eher unterhaltenden Wert hatte. ${ }^{\text {Ioo }}$ Gleichzeitig sind seine Bedenken ein gewichtiges Zeugnis dafür, dass der Bezug auf die Perserkriege kaum Teil einer ,offiziellen` Ideologie der Kaiserzeit gewesen sein

98 Lukian, Werke in drei Bänden. Hrsg. v. Jürgen Werner u. Herbert Greiner-Mai. Berlin I974, 2. Aufl. I98I, Bd. 3, 226 .

99 Plut. mor. 8I4 A-C = Praecepta gerendae reipublicae I7. Gleichzeitig wird deutlich, wie sehr es sich bei diesem ,Erinnern` um ein Elitenphänomen handelt, ein ,Erinnern', das freilich nur die griechischsprachigen Führungsschichten bestimmter Poleis, allen voran Athen, besonders pflegten. Siehe dazu Jung, Marathon (wie Anm. 30), 205-224.

Ioo Almagor, Empire (wie Anm. 26), 340-342, möchte darin gemeinsam mit anderen Zeugnissen einen Hinweis darauf erkennen, dass bei nicht wenigen der gebildeten griechischsprachigen Eliten die römische Herrschaft tatsächlich als bedrückend und die eigene Gegenwart somit analog zu den Verhältnissen unmittelbar nach dem Antalkidasfrieden erlebt wurde. Insofern käme dem Aufgreifen des Weltreichschemas, das die ,Endlichkeit‘ der jeweiligen Reiche betont, auch ein kritisches Potential zu; vgl. Makhlaiuk, Memory (wie Anm. 26), 302-304. 
kann, sonst hätte sich Plutarch wohl nicht veranlasst gesehen, auf die damit einhergehenden Gefahren aufmerksam zu machen. ${ }^{\text {ror }}$

Freilich kann man diskutieren, ob Äußerungen wie jene Plutarchs vielleicht bereits eine neue Haltung gegenüber dem Narrativ der Perserkriege manifestieren und dafür den Partherfeldzug Trajans verantwortlich machen. ${ }^{\text {I02 }}$ Tatsächlich hat Antony Spawforth so argumentiert und eine deutlich veränderte Positionierung Roms gegenüber den Perserkriegen im 2.Jahrhundert n.Chr. ausgemacht. Spätestens in dieser Zeit seien die Parther nicht mehr als Bedrohung gesehen worden, wodurch der Vergleich der römischen Kaiser mit Alexander dem Großen an Attraktivität gewonnen habe. Demgegenüber sei die Bedeutung der Perserkriege verblasst. ${ }^{\text {I03 }}$

Dieses Bild ist in jüngerer Zeit noch etwas geschärft worden. Eingebettet in das von Rom seit dem I.Jahrhundert v.Chr. gepflegte Partherbild ist Charlotte Lerouge auch der damit einhergehenden Instrumentalisierung der Perserkriege nachgegangen. ${ }^{\text {I04 }}$ Während in der späten Republik noch das Ideal der universellen Weltherrschaft und damit das Vorbild Alexanders von Pompeius, Caesar und Marc Anton gepflegt worden sei ${ }^{\text {105 }}$, habe Augustus damit begonnen, auch die Perserkriege als Mittel der offiziellen Propaganda einzusetzen ${ }^{106}$. Dabei sei das Partherreich als faktisch besiegter Feind und damit als römisches Klientelkönigtum präsentiert worden. Diese Fiktion sei bis zu den Flaviern weiter gepflegt worden, wobei man gleichzeitig alle militärischen Eroberungspläne de facto aufgegeben habe. ${ }^{107}$ Erst Trajan habe dann versucht, Anspruch und Wirklichkeit durch seinen großangelegten Feldzug in Ein-

Ior Dion erinnert die Rhodier daran, dass es für sie - wie wohl für alle Griechen - jetzt nicht mehr möglich sei, ihre Tüchtigkeit auf ,allen‘ Gebieten zu beweisen: Dion Chrys. 3 I, I6 I-I62.

I02 Interessanterweise erwähnt Plutarch in diesem Zusammenhang nicht ausdrücklich die Thermopylen. Dies ist deshalb bemerkenswert, weil er in seinen beiden Schriften De malignitate Herodoti und Apopthegmata Laconica nicht nur Herodot für seine angeblich ,tendenziöse‘ Berichterstattung in die Kritik nimmt, sondern eben auch den „exemplarischen Heroismus“ des Leonidas und seiner Mitstreiter hervorhebt; Albertz, Heldentum (wie Anm. I7), I04-I Io. Vielleicht ging es Plutarch dabei aber in erster Linie darum, die von Herodot zu wenig gewürdigten Thebaner „zu ihrem historischen Recht kommen zu lassen“ (ebd. Io9). Darüber hinaus mögen dem „Gleichheitsbeauftragten für Griechen“ (so die Charakterisierung Plutarchs durch Baltrusch, Historia [wie Anm. 84], 27 I) andere Ereignisse der ,heroischen' griechischen Vergangenheit wichtiger gewesen sein.

I03 Spawforth, Unity (wie Anm. 26), 242.

I04 Lerouge, Parthes (wie Anm. 26).

I05 Ebd.9I-93.

I06 Ebd.I24-I29.

I07 Ebd.I46-I49. 
klang zu bringen. Damit habe sich erneut und nun endgültig der Vergleich mit Alexander in den Vordergrund gedrängt, und der Bezug auf die Perserkriege sei ins Hintertreffen geraten. ${ }^{108}$ Trotz des letztendlichen Scheiterns des Trajanfeldzuges hätten die Parther von nun an als besiegt gegolten, wobei man die römische Sieghaftigkeit durch ständige Kampagnen gegen den östlichen Nachbarn immer wieder in Erinnerung gerufen habe. Dies sei zu einem wichtigen Bestandteil kaiserlicher Legitimierungsstrategien geworden. ${ }^{\text {rog }}$

\section{I08 Ebd.I63-I69.}

Io9 Wenn Lucius Verus und Caracalla auf ihren Partherfeldzügen ein Kontingent peloponnesischer Hilfstruppen mit sich führen, ist das sicherlich eine Reminiszenz an die Perserkriege; Herodian. 4,8,3; Spawforth, Unity (wie Anm. 26), 239; Cartledge/Spawforth, Sparta (wie Anm.75), I9I; Lerouge, Parthes (wie Anm. 26), I63. Von diesen Soldaten sind mehrere Grabinschriften aus Sparta erhalten, wozu in einem Fall auch ein Relief gehört, das einen spartanischen Auxiliarsoldaten mit Keule und arkadischem Pilos zeigt; Paul Wolters, Ein Denkmal der Partherkriege, in: MDAI(A) 28, I903, 29I-300. Es bleibt jedoch die Frage, ob man damit ein offizielles Ideologem fasst, das die beiden kaiserlichen Feldzüge als „Perserkriege“ vorstellt, oder ob man in den spartanisch-peloponnesischen Truppen nicht eine Art Talisman sehen kann, der dem Feldzug Glück verheißen soll. Wie die Ausrüstung des spartanischen Legionärs mit Keule und Pilos zeigt, war damit nicht an eine ,historische' Verknüpfung mit den spartanischen Hopliten von Platäa o.ä. gedacht, sondern man hatte eher einen romantischen Anklang an vergangene Zeiten im Auge, den Wolters, Partherkriege, 297, nicht unzutreffend als einen Versuch charakterisierte, „die große Vergangenheit nachäffend wieder zu beleben, mit stolzen, jetzt bombastisch klingenden Namen“. Auf jeden Fall konnte es nicht schaden, auch andere ,historische 'Glücksbringer bei sich zu haben. So führte Caracalla auch ein Kontingent von Makedonen mit sich, das im Stile der Phalanx Alexanders des Großen ausgerüstet war; Cartledge/Spawforth, Sparta (wie Anm.75), I I8f. Hier passte der historische Vergleich wohl wesentlich besser, wie wir ja auch von entsprechenden Bemühungen Caracallas wissen, sich als neuer Alexander zu stilisieren; vgl. Sabine Müller, Bei Austreten Mord. Caracallas Tod im Partherkrieg, in: Christian Hoffstadt/Melanie Möller/Sabine Müller (Hrsg.), Mord und Totlach. Bochum/Freiburg im Breisgau 20 I4, I I-24. Schwieriger einzuschätzen ist, wenn Gordian III. 235 n. Chr. in Rom einen Agon zu Ehren der Athena Promachos ausrichtet, bevor er zu seinem Feldzug gegen die Sasaniden aufbricht: Spawforth, Unity (wie Anm. 26), 239f., mit Verweis auf Louis Robert, Deux concours grecs à Rome, in: Comptes rendus des séances de l'Académie des Inscriptions et Belles-Lettres I I 4, I 970, I I-I 7. Nach dem Zeugnis des „Chronographen von 354“ und mehrerer Inschriften, die die Existenz des penteterischen Agons bis in die Zeit Konstantins belegen, ist der Konnex zwischen Stiftung und Partherfeldzug nicht von der Hand zu weisen („Gordianus [...] agonem Minervae instituit. Excessit finibus Parthiae“). Wie eine nach dem erfolgreichen Feldzug in Ephesos gesetzte Inschrift bezeugt, war die offizielle Ideologie in erster Linie bemüht, den Kaiser als ,Weltenherrscher‘ zu präsentieren: tòv

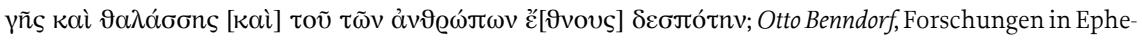
sos. Bd. I. Wien I 906, 2 I of., Nr. 2. Ähnliches gilt ja auch für Augustus; Schuler, Augustus (wie Anm. 86). Will man Gordians Stiftung als bewussten propagandistischen Akt verstehen, kann dies wohl nur vor der einmaligen Bedrohungslage durch die junge Dynastie der Sasaniden verstanden werden; Peter Heather, The Fall of the Roman Empire. A New History. London 2005, 6of. Andererseits mag man sich durchaus fragen, wer die Verknüpfung,Minerva (Athena Promachos) - Marathon - Partherfeldzug ' wirklich verstanden hat 
Ganz gleich ob man den Bezug auf die Perserkriege affirmativ als Teil der von den römischen Kaisern gepflegten ,offiziellen‘ Propaganda sieht oder dies eher kritisch betrachtet, so wird doch deutlich, dass sich mit dem beginnenden 2.Jahrhundert n. Chr. ideologische Veränderungen bemerkbar machen, von denen auch die Instrumentalisierung der Perserkriege nicht verschont blieb. Deren Bedeutung für die Gegenwart wurde deutlich (weiter) relativiert, und anstatt dessen wurden die Leistungen Roms stärker in den Vordergrund gerückt.

Ein für unsere Belange in jeder Hinsicht aufschlussreiches Zeugnis für diese Entwicklung stellen die Epitomae des Annius Florus dar. Das gemeinhin in die späte Regierungszeit Hadrians datierte Geschichtswerk ${ }^{\text {II }}$ kommt nicht besonders oft auf die Perserkriege zu sprechen. Einmal wird der Vergleich zwischen den Thermopylen und der von M. Calpurnius Flamma während des Ersten Punischen Krieges vollbrachten Heldentat bemüht, bei der der Militärtribun mit 300 Mann die Karthager so lange aufgehalten habe, bis das römische Heer sich absetzen konnte. Dabei habe Calpurnius letztlich Leonidas übertroffen, weil er das heldenhafte Unternehmen im Gegensatz zum Spartanerkönig überlebte. ${ }^{\text {II I }}$ Sextus Pompeius wird ein anderes Mal mit Xerxes gleichgesetzt, da sich beide durch schändliche Flucht ausgezeichnet hätten. ${ }^{\text {II2 }}$ Darüber hinaus hat der Perserkönig einen wesentlich prägnanteren Auftritt, in dem neben seinem Vater Dareios auch die Thermopylen und die Schlacht von Salamis Erwähnung finden. ${ }^{\text {II3 }}$ Insgesamt handelt es sich dabei um die umfangreichste

und welche Mobilisierungskraft dieser Inszenierung innewohnte. Letzteres gilt vor allem auch für die Frage der Bedeutung des Agons, nachdem Gordians III. Feldzug nicht unbedingt ein Erfolg gewesen und er dabei ums Leben gekommen war.

I Io Klaus Sallmann, § 462 O. (L.?) Annius Florus, in: ders. (Hrsg.), Die Literatur des Umbruchs. Von der römischen zur christlichen Literatur I I 7 bis 284 n. Chr. (Handbuch der Lateinischen Literatur der Antike, Bd. 4.) München I997, 329.

I I Flor. epit. I, I8,I3f.; vgl. dazu Albertz, Heldentum (wie Anm. I 7), 94f. mit Anm.5, die zugleich darauf hinweist, dass dieser Tribun bei M. Porcius Cato (Cato FRH 3 F 4,7a), der ersten Erwähnung dieser Tat sowie der Thermopylen in der römischen Literatur, noch anonym ist; zur Zahl 300 und der Wirkkraft dieser Tradition vgl. Kai Ruffing, 300, in: Boris Dunsch/Kai Ruffing (Hrsg.), Herodots Quellen - die Quellen Herodots. (Classica et Orientalia, Bd. 6.) Wiesbaden 2013, 20I-22 I; John Marincola, History without Malice? Herodotus Rewrites the Persian Wars, in: Jessica Priestly/Vasiliki Zali (Eds.), Brill's Companion to the Reception of Antiquity and Beyond. Leiden 20I6, IOI-I I9; John Marincola, The Historian as Hero. Herodotus and the 300 at Thermopylae, in: TAPhA I46, 20I6, 2 I 9-236; Wido Sieberer, I 809 oder die Schlacht bei den ,Thermopylen` Tirols, in: Michael Kasper/Robert Rollinger/Andreas Rudigier (Hrsg.), Sterben in den Bergen. (Montafoner Gipfeltreffen, 3.) Wien/Köln 2018 (im Druck).

I 2 Flor. epit. 2,I 8,9.

II3 Flor. epit. I,24. 
Reminiszenz der Perserkriege im Geschichtswerk des Florus. Dies geschieht im Kontext der Beschreibung des Syrischen Krieges gegen Antiochos III., wodurch sich plötzlich eine schlagende Parallele zu Herennius Dexippus ergibt. Dieser Krieg wird als einer der „furchterregendsten“ in der römischen Geschichte charakterisiert (non aliud formidolosius fama bellum fuit), und zwar aus folgendem Grund:

„weil die Römer ja an die Perser und den Osten, Xerxes und Dareios dachten, als gemeldet wurde, die unwegsamen Berge seien durchbohrt und das Meer mit Segeln bedeckt. Zudem versetzten die Drohungen der Götter die Menschen in Angst und Schrecken, als die Statue des Apollon in Cumae ununterbrochen schwitzte. Aber dies war die Furcht der Gottheit, die zu ihrem geliebten Asien hielt.“ “I4

Auch wenn von Antiochos III. nichts Vergleichbares mit der von Xerxes intendierten Errichtung des Athos-Kanals überliefert ist ${ }^{\mathrm{II} 5}$, so ist die Gleichsetzung zwischen den Seleukiden und Persern doch auffällig. Beide repräsentieren Asien, was auch durch die Bezeichnung des Krieges als bellum Asiaticum zum Ausdruck kommt. ${ }^{\text {II6 }}$ Antiochos steht für Asien, diese Gleichsetzung wird in der Folge mehrfach bemüht. So habe der Seleukidenkönig in seinem auf der Insel Euböa errichteten Feldlager „goldene und seidene Zelte“ aufstellen lassen (positis aureis sericisque tentoriis), habe mit Blas- und Streichinstrumenten aufspielen sowie, trotz des Winters, unzählige Rosen herbeischaffen lassen. ${ }^{I 17}$ Bereits hier wird sich der geschichtskundige Leser gedacht haben, was Florus gleich im Folgenden expressis verbis ausführt:

„Einen derartigen König also, der bereits durch seine eigene Verschwendung niedergeworfen war [iam luxuria sua debellatum], griff das römische Volk unter dem Konsuln M. Acilius Glabrio auf der Insel [Euböa] an; allein die Nachricht seiner Ankunft zwang ihn sofort, von der Insel zu fliehen. Dann holte er den

II 4 Flor. epit. I,24,2f.; dt. Übersetzung nach: Florus, Römische Geschichte. Eingel., übers. u. kommentiert v. Günter Laser. Darmstadt 2005, 97.

II 5 Xerxes' Durchbruch durch den Athos galt als paradigmatisch für das hybride Verhalten des Großkönigs und wurde meist in einem Atemzug mit dessen Schiffsbrücke genannt; Makhlaiuk, Memory (wie Anm. 26), 307 f.; Almagor, Empire (wie Anm. 26), 333. Vgl. Ampelius, Liber memorialis I3,4, der eine Art ,Zusammenschau des Bildungswissens seiner Zeit bietet. Dass hinter solchen Maßnahmen des Großkönigs ein Anspruch steckte, der ihn als Beherrscher der Elemente vorführte, ein Anspruch, den durchaus auch römische Kaiser für sich propagierten (Coleman, Launching into History [wie Anm. 39], 63, 66 f., 69, 74; Hardie, Images [wie Anm. 26], I 34f.; Almagor, Empire [wie Anm. 26], 34I), ist eine andere Sache.

II6 Flor. epit. I,24,8.

II7 Flor. epit. I,24,9. Zum, asiatischen` Luxus vgl. Makhlaiuk, Memory (wie Anm. 26), 312-315. 
kopfüber Flüchtenden an den Thermopylen ein, einem Ort, der durch das imponierende Gemetzel an den dreihundert Spartanern denkwürdig ist [locum trecentorum Laconum speciosa caede memorandum], - aber nicht einmal dort besaß Antiochos zuversichtlichen Glauben an die strategische Gunst dieses Ortes, Widerstand leisten zu können - und zwang ihn, zu Lande und zu Wasser zurückzuweichen. " ${ }^{118}$

Der Vergleich mit einer quasi gleichzeitig zu Lande und zu Wasser geführten Doppelschlacht nimmt deutliche Anleihe beim Narrativ Herodots, der die Schlachten an den Thermopylen und am Kap Artemision (sowie jene von Salamis und Himera, bzw. Platäa und Mykale) jeweils am gleichen Tag stattfinden ließ. ${ }^{\text {I } 9}$ Schließlich verfolgen die Römer die flüchtenden Truppen des Antiochos nach Asien, wo es bei Magnesia am Sipylos zur Entscheidungsschlacht kommt. Es überrascht nicht, dass sich der Seleukide dort mit „einer unbeschreiblich großen Menge an Hilfstruppen und regulären Truppen“ aufstellte (Hic rex incredibile dictu quibus auxiliis, quibus copiis consederat) - es sollen „300000 Fußsoldaten und eine an Reitern und sichelbewehrten Streitwagen nicht geringe Anzahl“ (trecenta milia peditum, equitum falcatorumque currum non minor numerus) gewesen sein. ${ }^{\mathrm{I} 20}$ Dazu kamen noch Elefanten „von unermesslicher Größe“ (elephantis ad hoc immensae magnitudinis). Besonderes Interesse verdient aber die Bewaffnung des feindlichen Heeres. Die Truppen seien nämlich mit „persischen Bögen“ (Persici arcus) ausgestattet gewesen. Diese seien allerdings durch heftige Regengüsse unbrauchbar geworden, was schließlich den überwältigenden römischen Sieg befördert habe. ${ }^{\text {I2I }}$

Mag der Leser bisher von einer gleichrangigen Parallelisierung der Perserkriege mit den von den Römern geleisteten Heldentaten gegen Antiochos III. ausgegangen sein, so bietet Florus unmittelbar nach seiner Schilderung der Thermopylenschlacht und des angeblich gleichzeitigen Seegefechts einen aufschlussreichen Einschub, der für die richtige Einordnung der historischen Vergleichsbeispiele sorgt:

I 8 Flor. epit. I, 24, Iof.; dt. Übersetzung nach: Florus, Römische Geschichte (wie Anm. I I 4), 99.

I 9 Reinhold Bichler, Der Synchronismus von Himera und Salamis. Eine quellenkritische Studie zu Herodot, in: Ekkehard Weber/Gerhard Dobesch (Hrsg.), Römische Geschichte, Altertumskunde und Epigraphik. Festschrift Artur Betz. Wien I985, 59-74 = Reinhold Bichler, Historiographie - Ethnographie - Utopie. Gesammelte Schriften, T. I: Studien zu Herodots Kunst der Historie. (Philippika. Marburger altertumskundliche Abhandlungen, I8/I.) Wiesbaden 2007, 47-54.

I 20 Flor. epit. I,24,I5f.

I 2 I Flor. epit. I,24,I7. 
„Athen soll nicht selbstzufrieden sein: In der Gestalt des Antiochos haben wir

Xerxes besiegt, in Aemilius sind wir mit Alkibiades gleichgezogen, mit Ephesos haben wir Salamis aufgewogen. “122

Hier wird nicht nur über die Perserkriege hinaus weiter ausgeholt - mit dem Verweis auf Alkibiades bis tief in den Peloponnesischen Krieg -, sondern auch der vor allem auf Athen gemünzte und wohl auch vor allem von Athen bemühte Vergleich mit den Perserkriegen in seiner Bedeutung zurechtgestutzt. Die Botschaft ist klar: Die römischen Erfolge sind den athenischen mindestens gleichwertig. Durch die damit einhergehende Maßregelung Athens (Ne sibi placeant Athenae) wird ein Ton der Zurückweisung angeschlagen, der Athen aus dem Rampenlicht verweist und Rom auf die hell erleuchtete Bühne stellt. Dadurch ergibt sich eine klare Rangordnung, die die römischen Erfolge als deutlich überlegen präsentiert. Dass Florus' Vergleich dabei hinkt, ist eine andere Sache. Denn weder kann von einer Gleichzeitigkeit der zwischen den Römern und den Seleukiden ausgefochtenen Land- und Seeschlacht gesprochen werden, noch lassen sich die von Alkibiades als Kommandeur der athenischen Flotte erzielten Siege bei Abydos und Kyzikos mit den Manövern der von L. Aemilius Regillus geführten römischen Flotte vergleichen, die schließlich der seleukidischen Armada bei Myonnesos in der Nähe von Ephesos eine vernichtende Niederlage beibrachte. ${ }^{\mathrm{I} 23}$ Aber darauf kommt es nicht an. Wichtig sind vielmehr die deutliche Relativierung der Bedeutung der Perserkriege und die gleichzeitige Aufwertung des Syrischen Krieges zu einer alles andere in den Schatten stellenden Konfrontation mit Asien, wobei die Auseinandersetzung bei den Thermopylen den Ausgangspunkt für den historischen Vergleich und die Hierarchisierung der Ereignisse zugunsten Roms darstellt. Damit fassen wir exakt jenes Geschichtsbild, das wir eingangs bei Herennius Dexippus kennengelernt haben und das sich somit bestens in einen größeren Zusammenhang einordnen lässt.

\footnotetext{
I22 Flor. epit. I,24,I3; dt. Übersetzung nach: Florus, Römische Geschichte (wie Anm. I I 4), 99.

I23 Die Seeschlacht unter dem Kommando des Aemilius wird von App. Syr. 27 als überwältigender römischer Erfolg präsentiert; Spawforth, Unity (wie Anm. 26), 243 mit Anm.40, hat dies mit dem kurz zuvor in App. Syr. 24 geschilderten Seegefecht verwechselt, ebenfalls bei Ephesos, wo von einer Niederlage des von Pausimachos geführten rhodischen Flottenverbandes gegen den seleukidischen Admiral Polyxenidas die Rede ist.
} 
IV.

Damit schließt sich der Bogen, wobei sich eine allerletzte Frage stellt. Im Gegensatz zu Florus hatte Herennius Dexippus ein griechischsprachiges Publikum vor Augen. Zwar mögen die oben zitierten mahnenden Worte Plutarchs sowie der allgemeine Stimmungswechsel in der historischen Einordnung der Perserkriege seit dem beginnenden 2.Jahrhundert n. Chr. auch auf den griechisch schreibenden Historiker nicht ohne Einfluss geblieben sein, doch lässt sich damit wirklich das in dem Wiener Dexippus-Fragment fassbare Geschichtsbild als das eines dem Zeitgeist gehorchenden griechischen Autors einordnen? Hier ist eine letzte, aber entscheidende Beobachtung vonnöten, um zu einer letztendlich befriedigenden Erklärung des vorliegenden Sachverhalts zu gelangen.

Erinnern wir uns daran, dass im Wiener Dexippus-Fragment eigentlich nicht der Autor, sondern vielmehr der Römer und Statthalter Achaeas Marianus zu seinen griechischen Soldaten spricht. Damit vermittelt die Stelle eine authentische Demonstration jenes von den römischen Eliten getragenen Geschichtsbildes, wie es spätestens seit dem Partherkrieg Trajans üblich geworden war. Dieses relativiert die Perserkriege in ihrer Bedeutung für die Gegenwart und bemüht gleichzeitig die herausragende Rolle des gegen Antiochos III. geführten Syrischen Krieges. Macht man sich die Vorstellung zu eigen, dass Herennius Dexippus seinen Marianus ein authentisches römisches Geschichtsbild seiner Zeit präsentieren lässt, so könnte es durchaus kein Zufall sein, dass er mit dem Böotarchen Dexippus und dem Athener Philostratos gerade zwei griechische Kommandanten nennt, deren Herkunftsorte sich bestens in die Gedankenwelt des Sprechers einordnen lassen. Man könnte dahinter sogar einen literarischen Kniff des Herennius Dexippus vermuten, der dazu diente, die von Marianus präsentierte Hierarchisierung bzw. Relativierung der historischen Ereignisse zu verstärken und somit dem Leser noch deutlicher vor Augen zu führen. ${ }^{\text {I24 }}$ So wird man sich daran erinnert haben, dass Athen zur Zeit des Syri-

I24 Für einen literarischen Kniff ließe sich vielleicht noch ein zusätzliches Argument anführen. Wie bereits eingangs angesprochen, war in den erhaltenen Abschnitten des Palimpsests schon einmal die Rede von der Errichtung einer Verteidigungsstellung bei den Thermopylen durch ein Volksaufgebot (f. I94 ${ }^{\mathrm{r}}$ Z. I-I6; Grusková/Martin, Neues Textstück [wie Anm. I], 32f.). Bei diesen wohl in das Jahr 250 n.Chr. zu datierenden Anstrengungen (ebd.30, 37-43; Schropp/Rollinger, Exercitus Romanus [wie Anm. I]) werden allerdings nicht nur Athener und Böoter, sondern auch Spartaner als Teile jenes Aufgebots genannt, das die Sperrstellung zu besetzen hatte. Auch werden in diesem Zusammenhang keine Anführer präsentiert. 
schen Krieges auf der Seite des Seleukiden und Theben zur Zeit des Perserkrieges auf jener des Großkönigs gekämpft hatte. Damit erhält das von Marianus bemühte Geschichtsbild eine zusätzliche Spitze, die die von den Griechen reklamierten Leistungen ihrer großen Geschichte im Vergleich zu den römischen Errungenschaften deutlich einschränkt. Auch der vermeintlich farblose Lamische Krieg fügt sich plötzlich bestens in diesen Kontext ein, kämpften dort doch die Böoter auf makedonischer Seite! ${ }^{125}$ Damit ergibt sich unversehens ein aufschlussreicher Befund, standen doch bei allen Vergleichsbeispielen die von den beiden griechischen Kommandeuren geführten Kontingente jeweils auf der ,falschen‘ Seite. Dies macht einmal mehr deutlich, dass nur unter römischer Führung ein militärischer Erfolg als gesichert gelten kann. Ob diese von Marianus geäußerte Auffassung auch jene des Herennius Dexippus ist, darf man vielleicht bezweifeln. ${ }^{\text {I26 }}$ Auf jeden Fall stattete Herennius Dexippus seinen Marianus mit einem authentischen Narrativ und Setting aus, das den ,griechischen 'Stolz auf die heroischen Leistungen der eigenen Vergangenheit mit einer unverkennbar kritischen Note versah. ${ }^{127} \mathrm{Ob}$ damit, das heißt

\footnotetext{
I25 Jehne, Koine Eirene (wie Anm. 23), 264.

I26 Die Beantwortung dieser Frage hängt davon ab, wie wir die Geschichtsauffassung des Dexipp rekonstruieren. Sieht man in Dexipp einen hellenozentrischen Autor (so etwa Piso, Bemerkungen [wie Anm. I], 208f.; vgl. oben Anm. I2), wird man die Auffassung vertreten, die Rede des Marianus hätte dem Weltbild des Autors stark widersprochen. Sieht man hinter dem Werk allerdings einen differenziert argumentierenden Autor, der bemüht ist, im Rahmen einer Geschichte des Gotenkrieges jene Faktoren zu bestimmen, die über Erfolg und Misserfolg im Krieg entscheiden, sieht die Sachlage wieder ganz anders aus. So hat Martin, Struktur (wie Anm. I), I08-I I4, deutlich gemacht, dass Dexipp im I. Buch seiner Skythika Kaiser Decius zwar als eine tragisch hilflose Figur schildere, die den eigenen Ansprüchen nicht gerecht werde und für ein Scheitern der römischen Zentralgewalt stehe, doch folge darauf in den letzten beiden Büchern eine Serie von römischen Siegen, die einen anderen Blick des Autors offenbarten. Damit sei das Werk „nicht hellenozentrisch und schon gar nicht primär eine Glorifizierung des allein wirksamen Widerstands der (vornehmlich griechischen) Poleis gegen die Barbaren“. Vielmehr handle es sich um „die Geschichte, wie sich die östliche Reichshälfte nach einer katastrophalen Niederlage langsam wieder erholt hat, dank des Einsatzes aller Teile der Bevölkerung und der Reichsführung, teilweise separat, teilweise in Kooperation“ (ebd. I I3). Damit wäre die Auffassung des Marianus gar nicht so weit weg von jener des Autors, auch wenn sie vielleicht bewusst überspitzt präsentiert wird; vgl. dazu auch Schropp/Rollinger, Exercitus Romanus (wie Anm. I), wo Dexipps Bild von Kaiser Decius weiter differenziert wird. Freilich spielt es in diesem Zusammenhang wohl auch eine Rolle, wann die im Codex Vindobonensis historicus gr. 73, ff. $192^{\mathrm{v}}-\mathrm{I} 93^{\mathrm{r}}$ geschilderten Ereignisse datiert werden (vgl. oben Anm.3). Platziert man sie ins Jahr 254/55 n. Chr., sind sie unmittelbar nach der ,durchwachsenen' Regierungszeit des Decius eingeordnet, setzt man sie hingegen in das Jahr 262 n. Chr., offenbart der historische Kontext bereits deutlicher die ,Erholungsphase‘ und den sich abzeichnenden Erfolg im Kampf gegen die Barbaren.

I27 Dass dies im Rahmen einer direkten Rede geschah, weist Dexippus einmal mehr als einen Epigonen
} 
durch die Betonung eines einer bestimmten Perspektive verpflichteten Blicks und der hiermit verbundenen Relativierung des ,Historischen', auch eine gewisse Kritik gegenüber den inhärenten Problemen eines jeden historischen Vergleichs und dessen Instrumentalisierung intendiert war, mag man in der Tat erwägen. Marianus wäre das wohl nicht in den Sinn gekommen, Herennius - und seinem Publikum vielleicht sehr wohl!

\section{Zusammenfassung}

Der Beitrag setzt sich kritisch mit der Bedeutung des Narratives „Perserkriege“ im Imperium Romanum vom I. bis 3.Jahrhundert n. Chr. auseinander. Ausgangspunkt der Überlegungen bildet ein erst kürzlich entdecktes Fragment des am Ende des 3. Jahrhunderts n.Chr. schreibenden Herennius Dexippus, das ausführlich diskutiert und in einen größeren historischen Kontext gestellt wird. Dabei wird deutlich, dass die Perserkriege zwar auch im Imperium eine breite literarische Rezeption erfuhren, dass sie jedoch nicht jene Bedeutung in der ,offiziellen' Reichsideolgie hatten, die ihnen von der modernen Forschung gemeinhin zugeschrieben wird. Zwar mögen griechische Intellektuelle, die stark auf Athen fokussiert waren, die Auseinandersetzung mit den Parthern und Sasaniden im Kontext der Perserkriege nach wie vor als eine ständige Konfrontation zwischen Asien und Europa gesehen haben, doch entsprach dies keineswegs einem von ,offiziellen' Stellen verbreiteten Deutungsmuster. Diese griffen zur Legitimation ihrer Politik vielmehr auf andere historische Ereignisse zurück, denen als Bezugspunkt eine wesentlich größere Rolle zukam. Neben dem Alexanderfeldzug scheint dabei der Syrische Krieg gegen Antiochos III., den „König Asiens“, eine besondere Rolle gespielt zu haben.

Prof. Dr. Robert Rollinger, Leopold-Franzens-Universität, Institut für Alte Geschichte und Altorientalistik, Am Langen Weg II, 6020 Innsbruck

des Thukydides aus (Mallan/Davenport, Gothic Invasions [wie Anm. I], 208), galten doch seit Thuk. I,22,I Reden als ein probates Mittel der Historiographie, um Authentizität zu erzeugen. 